\title{
Bidimensional non-parametric estimation of well-being distribution and poverty index
}

\author{
Youssou Ciss, Galaye Dia** and Aboubakary Diakhaby* \\ LERSTAD, UFR de Sciences Appliquées et de Technologie B.P. 234 Université Gaston Berger de \\ Saint-Louis, Sénégal
}

Received 10 September 2014; Accepted 22 November 2014

Copyright (c) 2014, Afrika Statistika. All rights reserved

\begin{abstract}
In this paper, we introduce a kernel-type version for the bi-dimensional extension of the Foster, Greer, and Thorbecke index that was introduced by Duclos et al. (2006a) for the purpose of a dominance approach to multidimensional poverty. The measure they used in their dominance exercise is essentially a generalization, from one to two dimensions, of the FGT index with separate poverty aversion parameters for each dimension. Our estimator is constructed by using a bidimensional Parzen-Rosenblatt kernel of a probability density function $(p d f)$. We next provide its complete asymptotic behaviour by establishing its almostsure uniform and its uniform mean square consistencies. A simulation study shows that it performs well for small samples comparatively to the empirical plug-in estimator. Our results are also extensions of those of Dia (2008) and of Ciss et al. (2014) in one dimension.
\end{abstract}

Résumé. Dans ce papier, nous proposons un estimateur pour la version bidimensionnelle de l'indice de pauvreté de Foster, Greer et Thorbecke, introduit par Duclos et al. (2006a) pour l'étude de pauvreté dans un cadre multidimensionnel grâce à la dominance stochastique. La mesure qu'ils utilisent dans cet exercice est en fait une extension bidimensionnelle de l'indice FGT avec deux paramètres pour l'aversion de la pauvreté, un dans chaque direction. Dans le processus de construction de notre estimateur, nous utilisons l'estimateur bidimensionnel de la densité de probabilité de Parzen-Rosenblatt. La convergence uniforme presque sûre et la convergence uniforme en erreur quadratique moyenne sont ensuite établies. Des simulations numériques montrent que notre estimateur se comporte bien, même pour les échantillons de petites tailles, face à l'estimateur empirique. Nos résultats constituent aussi une extension au cas bidimensionnel de ceux de Dia (2008) et de Ciss et al. (2014).

Key words: Bi-dimensional extension of the FGT; Poverty frontier; Parzen-Rosenblatt kernel in 2D; Uniform almost-surely consistency; Rate of convergence.

AMS 2010 Mathematics Subject Classification : 62G05; 91B82; 60F99.

*Corresponding author Aboubakary Diakhaby : diakhaby81@yahoo.fr, diakhaby@ugb.edu.sn

${ }^{* *}$ In memory, passed away in may 2013

Youssou Ciss : ciss.youssouf@gmail.com 


\section{Introduction}

Duclos et al. (2006a) considered a multidimensional extension of the FGT class of measures, to address robustness analysis of the choice of poverty indices and poverty lines. They used the dominance approach for poverty comparisons, as initially developed in Atkinson (1987), in Foster and Shorrocks (1988a), Foster and Shorrocks (1988b) and in Foster and Shorrocks (1988c). A major advantage of this approach is its ability in generating poverty orderings that are robust with respect to the determination of poverty lines. Then the sensivity of most of poverty measures to the poverty line makes this approach more important. Besides, it also ensures robustness with respect to the choice of a multidimensional poverty index over broad classes of them, as well as robustness over the manner in which multidimensional indicators interact between them, when describing overall individual well-being. Duclos et al. (2006b) also used the bivariate stochastic dominance techniques to investigate the incidence of poverty, measured in terms of household expenditures per capita and child height-for-age indicators.

Such important traits of this measure motivated us to have an asymptotic theory based on estimators constructed on random samples that would provide accurate approximations for small sizes. Their uniform asymptotic laws may lead to optimal choices of the two considered parameters. A number of different ways are possible to adopt, one of them is the use of the empirical plug-in estimator. We consider here, as a first study of such nature of this index, extensions in form of kernel-type statistics, that we already used in one-dimensional studies in Dia (2008), for $\alpha=0$ and $\alpha \geq 1$ and, by Ciss et al. (2014) for $\alpha \in] 0,1[$. By the way, the results exposed in these papers will be particular cases of results of the current paper. Our approach will demonstrate to give be better results than the one based on the empirical estimator.

To make the ideas more clear, let $x$ and $y$ be two indicators of individual well-being among, for example, income, expenditures, caloric consumption, life expectancy, height, body weight, extent of personal safety and freedom, etc. Throughout this paper $(X, Y)$ stands for the value of $(x, y)$ for a randomly selected individual of the population. Then $(X, Y)$ is a random couple of nonnegative real numbers defined of a given probability space $(\Omega, \mathcal{A}, \mathbb{P})$ whose cumulative distribution function $(c d f)$ is denoted by $F(.,$.$) and we suppose that it admits a p d f f(.,$.$) .$ From now all the expections are done with respect to this probability space.

The bi-dimensional extension of the FGT Foster et al. (1984) class of poverty measures by Duclos et al. (2006a) is denoted by $P\left(z_{1}, z_{2}, \alpha_{1}, \alpha_{2}\right)$ and is defined as follows, for $\left(\alpha_{1} \geq\right.$ $\left.0, \alpha_{2} \geq 0\right):$

$$
= \begin{cases}\int_{0}^{z_{1}} \int_{0}^{z_{2}}\left(\frac{z_{1}-x}{z_{1}}\right)^{\alpha_{1}}\left(\frac{z_{2}-y}{z_{2}}\right)^{\alpha_{2}} f(x, y) d x d y & \text { if } z_{1}>0 \text { and } z_{2}>0 \\ 0 & \text { otherwise }\end{cases}
$$

where $z_{1}$ (resp. $z_{2}$ ) represents the poverty line for the dimension $x$ (resp. $y$ ). This index is useful for ordinal robust comparisons of poverty, even when the measurements are made across the intersection of the two dimensions considered.

Now let us consider, for $n \geq 1$, a random sample $\left(X_{1}, Y_{1}\right), \ldots,\left(X_{n}, Y_{n}\right)$ from $(X, Y)$, defined of the probability space defined above. The empirical plug-in estimator of 1 is given by 


$$
\widehat{P}_{n}\left(z_{1}, z_{2}, \alpha_{1}, \alpha_{2}\right)=\frac{1}{n} \sum_{i=1}^{n} \sum_{j=1}^{n}\left(1-\frac{X_{i}}{z_{1}}\right)_{+}^{\alpha_{1}}\left(1-\frac{Y_{j}}{z_{2}}\right)_{+}^{\alpha_{2}}
$$

where $x_{+}=\max (0, x)$.

From there, we use the Parzen (1962) kernel estimator of the density $f(x, y)$ :

$$
\hat{f}(x, y)=\frac{1}{n} \sum_{i=1}^{n} \frac{1}{h_{1} h_{2}} K\left(\frac{x-X_{i}}{h_{1}}\right) K\left(\frac{y-Y_{i}}{h_{2}}\right) .
$$

Combining these two last facts, and based on Riemann sum, we are able to propose the following kernel estimator of the DSY index $1: P_{n}\left(z_{1}, z_{2}, \alpha_{1}, \alpha_{2}\right)$

$$
\left.=\frac{1}{n} \sum_{k=1}^{n} \sum_{i=0}^{\left[\frac{z_{1}}{h_{1}}\right]\left[\frac{z_{2}}{h_{2}}\right]} \sum_{j=0}^{i h_{1}}\right)^{\alpha_{1}}\left(1-\frac{j h_{2}}{z_{2}}\right)^{\alpha_{2}} K\left(\frac{X_{k}-i h_{1}}{h_{1}}\right) K\left(\frac{Y_{k}-i h_{2}}{h_{2}}\right),
$$

where $\left[\frac{\dot{h}}{h_{i}}\right]$, is the integer part of $\dot{\overline{h_{i}}}, i=1,2 \quad \alpha_{i} \geq 0, h_{i}=h_{i}(n), i=1,2$ are positive nonrandom sequences of real numbers tending to zero as as $n$ tends to infinity, and finally $K(x)$ is a Borel function satisfying the following hypotheses:

$$
\left(\mathbf{H}_{1}\right) \sup _{-\infty<x<+\infty}|K(x)|<+\infty,\left(\mathbf{H}_{\mathbf{2}}\right) \int_{\mathbb{R}} K(x) d x=1,\left(\mathbf{H}_{\mathbf{3}}\right) \lim _{x \rightarrow \pm \infty}|x||K(x)|=0 .
$$

For readibiliy's sake, we expose our construction of the latter statistic in the appendix section 5 .

As announced we are going to describe the complete asymptotic theory of (3). Our best achievement is establishing the almost-sure consitency with respect to the parameters $\left(\alpha_{1}, \alpha_{2}\right) \in\left(\mathbb{R}_{+}^{*}\right)^{2}$ as well as the uniform mean square efficiency. We have been able to conduct simulation studies that showed good performances for small sizes data, better that results from the empirical plug-in statistics.

The rest of the paper is organized as follows. In Section 2, will state full details of the results. In Section 3, we will provide simulation studies outcomes and relevant comments as well as a compairison with results from the empirical approach that was used until now. The complete proofs are then given in Section 4.

\section{Convergence of the estimator}

We will need a number of hypotheses and conditions for our theorems. We need to derive the following one $\mathbb{K}$,from the function $K$, defined on $\mathbb{R}^{2}$ by $\mathbb{K}(x, y)=K(x) K(y)$. This this latter inherits from $K$ these two properties : $\sup _{-\infty<x, y<+\infty}|\mathbb{K}(x, y)|<+\infty$ and

$$
\iint_{\mathbb{R}^{2}} \mathbb{K}(x, y) d x d y=1, \lim _{\|(x, y)\| \rightarrow \infty}\|(x, y)\||\mathbb{K}(x, y)|=0 .
$$

Now additional hypotheses on $\mathbb{K}$ or $K$ are the following :

$\left(\mathbf{H}_{4}\right) K$ is a bounded variation function on $\mathbb{R}$ and we denote by $V(\mathbb{R})$ its total variation. 
$\left(\mathbf{H}_{5}\right) \iint_{\mathbb{R}^{2}}|u v||\mathbb{K}(u, v)|<+\infty$.

$\left(\mathbf{H}_{6}\right)$ There exists a non increasing function $\lambda$ such that $\lambda\left(\frac{u}{h_{1}}, \frac{v}{h_{2}}\right)=O\left(h_{1} h_{2}\right)$ on any bounded rectangle and for two couple of real numbers $x=\left(x_{1}, x_{2}\right)$ and $y=\left(y_{1}, y_{2}\right)$,

$$
|\mathbb{K}(x)-\mathbb{K}(y)| \leq \lambda\|x-y\| \quad \text { and } \quad \lambda(u, v) \longrightarrow 0,(u, v) \rightarrow(0,0), u \geq 0, v \geq 0,
$$

where $\|\cdot\|$ stands for the Euclidean norm.

Finally, these condions depend of the $p d f f(x, y)$ :

$\mathbf{C}_{1}: f(x, y)$ is uniformly continuous.

$\mathbf{C}_{2}: f(x, y)$ admits almost everywhere derivative $f^{\prime}(x, y) \in L_{1}(\mathbb{R} \times \mathbb{R})$.

We are now able to describe our results that we organize in subsections.

\subsection{The uniform almost sure consistency and the behavior of the bias}

Theorem 1. Assume that the hypotheses $H_{4}$ and $C_{1}$ hold. Then, for all $b>0$ (i.e.b= $\left(b_{1}, b_{2}\right), b_{1}>0$ and $\left.b_{2}>0\right)$, the estimator $P_{n}\left(z_{1}, z_{2}, \alpha_{1}, \alpha_{2}\right)$ converges uniformly almost surely on $\left[0, b_{1}\right] \times\left[0, b_{2}\right]$ to $P\left(z_{1}, z_{2}, \alpha_{1}, \alpha_{2}\right)$ as $n \rightarrow+\infty$ i.e.

$$
P\left(\lim _{n \rightarrow+\infty} \sup _{\left(z_{1}, z_{2}\right) \in\left[0, b_{1}\right] \times\left[0, b_{2}\right]}\left|P_{n}\left(z_{1}, z_{2}, \alpha_{1}, \alpha_{2}\right)-P\left(z_{1}, z_{2}, \alpha_{1}, \alpha_{2}\right)\right|=0\right)=1,
$$

provided $n h_{1}^{2} h_{2}^{2}(\log \log n)^{-1} \rightarrow+\infty$ as $n \rightarrow+\infty$.

Theorem 2. Assume that the hypotheses $H_{4}, H_{5}$ and $C_{2}$ hold, for all $b>0 \quad(b=$ $\left(b_{1}, b_{2}\right)$, the estimator $P_{n}\left(z_{1}, z_{2}, \alpha_{1}, \alpha_{2}\right)$ converges uniformly almost surely on $\left[0, b_{1}\right] \times$ $\left[0, b_{2}\right]$ to $P\left(z_{1}, z_{2}, \alpha_{1}, \alpha_{2}\right)$ as $n \rightarrow+\infty$ i.e.

$$
P\left(\lim _{n \rightarrow+\infty} \sup _{\left(z_{1}, z_{2}\right) \in\left[0, b_{1}\right] \times\left[0, b_{2}\right]}\left|P_{n}\left(z_{1}, z_{2}, \alpha_{1}, \alpha_{2}\right)-P\left(z_{1}, z_{2}, \alpha_{1}, \alpha_{2}\right)\right|=0\right)=1,
$$

provided $n h_{1}^{2} h_{2}^{2}(\log \log n)^{-1} \rightarrow+\infty$ as $n \rightarrow+\infty$.

Lemma 1. If $C_{1}$ holds, then for all $b>0$, we have

$$
\lim _{n \rightarrow+\infty} \sup _{\left(z_{1}, z_{2}\right) \in\left[0, b_{1}\right] \times\left[0, b_{2}\right]}\left|\mathbb{E}\left(P_{n}\left(z_{1}, z_{2}, \alpha_{1}, \alpha_{2}\right)\right)-P\left(z_{1}, z_{2}, \alpha_{1}, \alpha_{2}\right)\right|=0 .
$$

Lemma 2. Let $M=\sup _{\left(z_{1}, z_{2}\right) \in \mathbb{R}^{2}} \frac{F\left(z_{1}, z_{2}\right)}{z_{1} z_{2}}$ and $A=\sup _{(x, y) \in \mathbb{R}^{2}} f(x, y)$, If $H_{5}$ and $C_{2}$ hold, then

$$
\begin{aligned}
\sup _{\left(z_{1}, z_{2}\right) \in \mathbb{R}^{2}}\left|\mathbb{E}\left(P_{n}\left(z_{1}, z_{2}, \alpha_{1}, \alpha_{2}\right)\right)-P\left(z_{1}, z_{2}, \alpha_{1}, \alpha_{2}\right)\right| & \leq h_{1} h_{2}\left(\left(\int_{\mathbb{R}^{2}}\left|f^{\prime}(x, y)\right| d x d y\right)\right. \\
& \times\left(\int_{\mathbb{R}^{2}}(|u|+1)(|v|+1)|\mathbb{K}(u, v)| d u d v\right) \\
& \left.+4\left(\alpha_{1} \alpha_{2} M+A h_{1} h_{2}\right) \int_{-\infty}^{+\infty}|\mathbb{K}(u, v)| d u d v\right) .
\end{aligned}
$$

Remark 1. If $K$ satisfies the hypothesis $H_{5}$, then by using $H_{1}$, the kernel $\hat{\mathbb{K}}=$ $\frac{\mathbb{K}^{2}}{\int_{\mathbb{R}^{2}} \mathbb{K}^{2}\left(y_{1}, y_{2}\right) d y_{1} d y_{2}} \quad$ also satisfies it. 
From the two previous lemmas, we get the following corollaries

Corollary 1. Under the assumptions of Lemma 1, we have uniformly on $\left[0, b_{1}\right] \times\left[0, b_{2}\right]$

$$
\begin{aligned}
& \lim _{n \rightarrow+\infty} \mathbb{E}\left(\sum_{i=1}^{\left[\frac{z_{1}}{h_{1}}\right]} \sum_{j=1}^{\left[\frac{z_{2}}{h_{2}}\right]}\left(1-\frac{i h_{1}}{z_{1}}\right)^{2 \alpha_{1}}\left(1-\frac{j h_{2}}{z_{2}}\right)^{2 \alpha_{2}} K^{2}\left(\frac{X_{k}-i h_{1}}{h_{1}}\right) K^{2}\left(\frac{Y_{k}-i h_{2}}{h_{2}}\right)\right) \\
& =P\left(z_{1}, z_{2}, 2 \alpha_{1}, 2 \alpha_{2}\right) \int_{\mathbb{R}^{2}} \mathbb{K}^{2}\left(y_{1}, y_{2}\right) d y_{1} d y_{2} .
\end{aligned}
$$

Corollary 2. If the assumptions of Theorem 2 hold and if $h_{1} h_{2}=O\left(n^{-1} \log \log n\right)^{1 / 4}$, then for all $b>0$, we have almost-surely

$$
\sup _{\left(z_{1}, z_{2}\right) \in\left[0, b_{1}\right] \times\left[0, b_{2}\right]}\left|P_{n}\left(z_{1}, z_{2}, \alpha_{1}, \alpha_{2}\right)-P\left(z_{1}, z_{2}, \alpha_{1}, \alpha_{2}\right)\right|=O\left(n^{-1} \log \log n\right)^{1 / 4} .
$$

\subsection{The uniform mean square consistency}

Theorem 3. If $H_{6}$ and $C_{1}$ hold. Then

1.

$$
\begin{aligned}
\lim _{n \rightarrow+\infty} n \operatorname{Var}\left(P_{n}\left(z_{1}, z_{2}, \alpha_{1}, \alpha_{2}\right)\right) & =P\left(z_{1}, z_{2}, 2 \alpha_{1}, 2 \alpha_{2}\right) \int_{\mathbb{R}^{2}} \mathbb{K}^{2}\left(y_{1}, y_{2}\right) d y_{1} d y_{2} \\
& -\left(P\left(z_{1}, z_{2}, \alpha_{1}, \alpha_{2}\right)\right)^{2} .
\end{aligned}
$$

2. For all $b>0$,

$$
\lim _{n \rightarrow+\infty} \sup _{\left(z_{1}, z_{2}\right) \in\left[0, b_{1}\right] \times\left[0, b_{2}\right]} \mathbb{E}\left(P_{n}\left(z_{1}, z_{2}, \alpha_{1}, \alpha_{2}\right)-P\left(z_{1}, z_{2}, \alpha_{1}, \alpha_{2}\right)\right)^{2}=0 .
$$

Theorem 4. Assume that $H_{6}$ and $C_{2}$ hold. Then

$$
\begin{aligned}
\lim _{n \rightarrow+\infty} n \operatorname{Var}\left(P_{n}\left(z_{1}, z_{2}, \alpha_{1}, \alpha_{2}\right)\right) & =P\left(z_{1}, z_{2}, 2 \alpha_{1}, 2 \alpha_{2}\right) \int_{\mathbb{R}^{2}} \mathbb{K}^{2}\left(y_{1}, y_{2}\right) d y_{1} d y_{2} \\
& -\left(P\left(z_{1}, z_{2}, \alpha_{1}, \alpha_{2}\right)\right)^{2} .
\end{aligned}
$$

Moreover, if $H_{5}$ holds we have for all $b>0$,

$$
\lim _{n \rightarrow+\infty} \sup _{\left(z_{1}, z_{2}\right) \in\left[0, b_{1}\right] \times\left[0, b_{2}\right]} \mathbb{E}\left(P_{n}\left(z_{1}, z_{2}, \alpha_{1}, \alpha_{2}\right)-P\left(z_{1}, z_{2}, \alpha_{1}, \alpha_{2}\right)\right)^{2}=0 .
$$

For the proof of this theorem, first we prove the Theorem 5 below using the

Lemma 3. Let $0 \leq \theta_{i}^{j} \leq 1, i, j=1,2$. Then for all $x=\left(x_{1}, x_{2}\right), y=\left(y_{1}, y_{2}\right)$ and $x \neq y$ we have

$$
\begin{aligned}
& \lim _{n \rightarrow+\infty} \sup _{\left(z_{1}, z_{2}\right) \in[0,1] \times[0,1]}\left(\left(h_{1} h_{2}\right)^{-2} \iint_{-\infty}^{+\infty} \mid K\left(\frac{u_{1}-x_{1}+\theta_{1}^{1}}{h_{1}}, \frac{u_{2}-x_{2}+\theta_{1}^{2}}{h_{2}}\right)\right. \\
& \left.\times K\left(\frac{u_{1}-y_{1}+\theta_{2}^{1}}{h_{1}}, \frac{u_{2}-y_{2}+\theta_{2}^{2}}{h_{2}}\right) \mid f\left(u_{1}, u_{2}\right) d u_{1} d u_{2}\right)=0 .
\end{aligned}
$$


Theorem 5. Assume that the hypothesis $H_{6}$ holds. Then for all $b>0$,

$$
\begin{aligned}
& \lim _{n \rightarrow+\infty} \sup _{\left(z_{1}, z_{2}\right) \in\left[0, b_{1}\right] \times\left[0, b_{2}\right]} \sum_{0 \leq i_{1} \neq j_{1} \leq\left[\frac{z_{1}}{h_{1}}\right]} \sum_{0 \leq i_{2} \neq j_{2} \leq\left[\frac{z_{2}}{h_{2}}\right]}\left(1-\frac{i_{1} h_{1}}{z_{1}}\right)^{\alpha_{1}}\left(1-\frac{j_{1} h_{1}}{z_{1}}\right)^{\alpha_{1}} \\
& \times\left(1-\frac{i_{2} h_{2}}{z_{2}}\right)^{\alpha_{2}}\left(1-\frac{j_{2} h_{2}}{z_{2}}\right)^{\alpha_{2}} \\
& \times \int_{\mathbb{R}^{2}} K\left(\frac{u_{1}-i_{1} h_{1}}{h_{1}}\right) K\left(\frac{u_{1}-j_{1} h_{1}}{h_{1}}\right) K\left(\frac{u_{2}-i_{2} h_{2}}{h_{2}}\right) \\
& K\left(\frac{u_{2}-j_{2} h_{2}}{h_{2}}\right) f\left(u_{1}, u_{2}\right) d u_{1} d u_{2}=0 .
\end{aligned}
$$

Remark 2. The estimator $P_{n}\left(z_{1}, z_{2}, \alpha_{1}, \alpha_{2}\right)$ has asymptotic efficiency with respect to $\widehat{P}_{n}\left(z_{1}, z_{2}, \alpha_{1}, \alpha_{2}\right)$,

$$
e\left(z_{1}, z_{2}, \alpha_{1}, \alpha_{2}\right)=\frac{P\left(z_{1}, z_{2}, 2 \alpha_{1}, 2 \alpha_{2}\right) \int_{\mathbf{R}^{2}} \mathbb{K}^{2}\left(y_{1}, y_{2}\right) d y_{1} d y_{2}-\left(P\left(z_{1}, z_{2}, \alpha_{1}, \alpha_{2}\right)\right)^{2}}{P\left(z_{1}, z_{2}, 2 \alpha_{1}, 2 \alpha_{2}\right)-\left(P\left(z_{1}, z_{2}, \alpha_{1}, \alpha_{2}\right)\right)^{2}}
$$

The integral $\int_{\mathbf{R}^{2}} K^{2}\left(y_{1}, y_{2}\right) d y_{1} d y_{2}$ is strictly inferior to 1 for $K$ product of two conventional kernels Parzen (1962) p.1068. Then we have in this case $e\left(z_{1}, z_{2}, \alpha_{1}, \alpha_{2}\right)<1$. In Theorem 4, the rate of convergence in mean square is estimated to $O\left(\frac{1}{n}\right)$ if $h_{1} h_{2}$ is estimated to $O\left(\frac{1}{\sqrt{n}}\right)$.

\section{Simulations}

We conducted simulation studies based on of 75 replications of samples of size $n=50$ and computed the value of our estimator and that of the empirical plug-in estimator. We considered Gaussian kernels that fullfil $H_{i}, i=1, \ldots, 6$ and $h_{j}=1 / \sqrt{n \log n}, j=1,2$. As for the distribution, we used a couple of independent coordinates with first margin following a Pareto distribution type on $[0,1]$ with parameters $x_{0}=0.02$ and $b=0.2$, and a second margin following an exponential distribution with parameter $\lambda=1$. For a fixed couple of poverty lines, for a fixed $n$, replications of samples give the following replicated values for the two estimators, our kernel-type one and the empirical plug-in estimator, in the respective replicated sequences :

$$
\left(P_{n, 1}\left(z_{1}, z_{2}, \alpha_{1}, \alpha_{2}\right), \ldots, P_{n, 75}\left(z_{1}, z_{2}, \alpha_{1}, \alpha_{2}\right)\right)
$$

and

$$
\left(\widehat{P}_{n, 1}\left(z_{1}, Z_{2}, \alpha_{1}, \alpha_{2}\right), \ldots, \widehat{P}_{n, 75}\left(z_{1}, z_{2}, \alpha_{1}, \alpha_{2}\right)\right)
$$

From each sequence, we compute the mean value $m v_{i}$, the mean square error $m s q_{i}$ and the variance $\sigma_{i}$, where $i=1$ corresponds to the Kernel-type estimator and $i=2$ to the empirical estimator. For the first case, this is :

$$
\begin{gathered}
m v_{1}=\overline{P_{n}\left(z_{1}, z_{2}, \alpha_{1}, \alpha_{2}\right)}=\frac{1}{75} \sum_{i=1}^{75} P_{n, i}\left(z_{1}, z_{2}, \alpha_{1}, \alpha_{2}\right), \\
m s q e 1=\frac{1}{75} \sum_{i=1}^{75}\left(P_{n, i}\left(z_{1}, z_{2}, \alpha_{1}, \alpha_{2}\right)-P\left(z_{1}, z_{2}, \alpha_{1}, \alpha_{2}\right)\right)^{2},
\end{gathered}
$$


Y. Ciss, G. Dia, A. Diakhaby, Afrika Statistika, Vol. 9, 2014, pages 695-725. Bidimensional non-parametric estimation of well-being distribution and poverty index.

$$
\sigma_{1}=\frac{1}{74} \sum_{i=1}^{75}\left(P_{n, i}\left(z_{1}, z_{2}, \alpha_{1}, \alpha_{2}\right)-\overline{P_{n}\left(z_{1}, z_{2}, \alpha_{1}, \alpha_{2}\right)}\right)^{2} .
$$

For the second case, we similarly define $m v_{2}, m s q e 2$ and $\sigma_{2}$.

Next, we choose $\left(\alpha_{1}, \alpha_{2}\right)=(0,0),\left(\alpha_{1}, \alpha_{2}\right)=(1,1)$ and $\left(\alpha_{1}, \alpha_{2}\right)=(2,2)$. The outcomes are summerized in the table below.

\begin{tabular}{|c|c|c|c|c|c|}
\hline$\left(z_{1}, z_{2}\right)$ & $(0.1,0.1)$ & $(0.1,0.4)$ & $(0.1,0.8)$ & $(0.4,0.4)$ & $(0.4,0.8)$ \\
\hline \multicolumn{6}{|c|}{$\left(\alpha_{1}=0, \alpha_{2}=0\right) ; n=50$} \\
\hline mqse1 & $4.52376 \mathrm{e}-04$ & $4.25607 \mathrm{e}-03$ & $1.07638 \mathrm{e}-02$ & $4.40944 \mathrm{e}-03$ & $1.03522 \mathrm{e}-02$ \\
\hline mqse2 & 0.871967 & 7.923746 & 23.95921 & 35.87961 & 104.3472 \\
\hline$\sigma_{1}$ & $9.91613 \mathrm{e}-06$ & $9.84891 \mathrm{e}-05$ & $2.46968 \mathrm{e}-04$ & $7.26561 \mathrm{e}-04$ & $1.5741 \mathrm{e}-03$ \\
\hline$\sigma_{2}$ & 0.264786 & 1.283177 & 3.634951 & 1.867318 & 23.41582 \\
\hline \multicolumn{6}{|c|}{$\left(\alpha_{1}=1, \alpha_{2}=1\right) ; n=50$} \\
\hline mqse1 & 2.486597 & 0.2256831 & 0.0739875 & 1.124062 & 0.342812 \\
\hline mqse2 & 1.993245 & 0.0701992 & 0.6961594 & 1.439354 & 10.39371 \\
\hline$\sigma_{1}$ & $6.54092 \mathrm{e}-08$ & $5.20152 \mathrm{e}-04$ & $5.26027 \mathrm{e}-06$ & $6.83591 \mathrm{e}-05$ & $1.80800 \mathrm{e}-04$ \\
\hline$\sigma_{2}$ & 0.0125571 & 0.0639057 & 0.641102 & 1.507185 & 4.193532 \\
\hline \multicolumn{6}{|c|}{$\left(\alpha_{1}=2, \alpha_{2}=2\right) ; n=50$} \\
\hline mqse1 & 1273.946 & 4.87621 & 0.168094 & 35.68207 & 0.342669 \\
\hline mqse2 & 1277.474 & 5.709271 & 0.507776 & 49.40013 & 4.335062 \\
\hline$\sigma_{1}$ & $4.31454 \mathrm{e}-10$ & $7.37444 \mathrm{e}-08$ & $2.16516 \mathrm{e}-07$ & $1.40352 \mathrm{e}-05$ & $4.40888 \mathrm{e}-05$ \\
\hline$\sigma_{2}$ & $1.40887 \mathrm{e}-03$ & $8.76890 \mathrm{e}-03$ & $2.32100 \mathrm{e}-02$ & 0.15877 & 0.323104 \\
\hline
\end{tabular}

Table 1. Comparative table of results simulations for small samples

The studied cases $P\left(z_{1}, z_{2}, 0,0\right), P\left(z_{1}, z_{2}, 1,1\right), P\left(z_{1}, z_{2}, 2,2\right)$ are commonly and respectively called the two-dimensional poverty rate intersection, the two-dimensional depth of poverty intersection and the two-dimensional severity of poverty intersection Duclos et al. (2006a). 
The results speak themselves. The kernel-type estimator behave much better.

\section{Proofs of the results.}

Lemma 4. Let $0 \leq \theta_{i}<1, \quad i=1,2$. If $f(x, y)$ is uniformly continuous and bounded, we have uniformly relatively to $\left(\theta_{i}, y_{i}\right) \subset[0,1] \times R$

$$
\begin{array}{r}
\lim _{n \rightarrow+\infty} \int_{\mathbb{R}^{2}}\left(h_{1, n} h_{2, n}\right)^{-1} \mathbb{K}\left(\frac{u_{1}}{h_{1, n}}, \frac{u_{2}}{h_{2, n}}\right) f\left(u_{1}+y_{1}-\theta_{1} h_{1, n}, u_{2}+y_{2}-\theta_{2} h_{2, n}\right) d u_{1} d u_{2} \\
=f\left(y_{1}, y_{2}\right) \int_{\mathbb{R}^{2}} \mathbb{K}\left(u_{1}, u_{2}\right) d u_{1} d u_{2} .
\end{array}
$$

Proof. Since $f(x, y)$ is uniformly continuous, then for $\varepsilon>0$, there exists $\eta>0$ such that for $\left\|\theta_{i} h_{i, n}\right\|<\eta, i=1,2$,

$$
\begin{array}{rl}
\mid \int_{\mathbb{R}^{2}}\left(h_{1, n} h_{2, n}\right)^{-1} & \mathbb{K}\left(\frac{u_{1}}{h_{1, n}}, \frac{u_{2}}{h_{2, n}}\right) f\left(u_{1}+y_{1}-\theta_{1} h_{1, n}, u_{2}+y_{2}-\theta_{2} h_{2, n}\right) d u_{1} d u_{2} \\
& -\int_{\mathbb{R}^{2}}\left(h_{1, n} h_{2, n}\right)^{-1} \mathbb{K}\left(\frac{u_{1}}{h_{1, n}}, \frac{u_{2}}{h_{2, n}}\right) f\left(u_{1}+y_{1}, u_{2}+y_{2}\right) d u_{1} d u_{2} \mid \\
& \leq \int_{\mathbb{R}^{2}}\left(h_{1, n} h_{2, n}\right)^{-1}\left|\mathbb{K}\left(\frac{u_{1}}{h_{1, n}}, \frac{u_{2}}{h_{2, n}}\right)\right| \mid f\left(u_{1}+y_{1}-\theta_{1} h_{1, n}, u_{2}+y_{2}-\theta_{2} h_{2, n}\right) \\
& -f\left(u_{1}+y_{1}, u_{2}+y_{2}\right)\left|d u_{1} d u_{2} \leq \varepsilon \int_{\mathbb{R}^{2}}\left(h_{1, n} h_{2, n}\right)^{-1}\right| \mathbb{K}\left(\frac{u_{1}}{h_{1, n}}, \frac{u_{2}}{h_{2, n}}\right) \mid d u_{1} d u_{2} .
\end{array}
$$

For $y=\left(y_{1}, y_{2}\right)$, choose then $n$ large enough such that $\left\|\theta h_{n}\right\|<\eta, \theta=\left(\theta_{1}, \theta_{2}\right)$ to get the inequality for all $\left(\theta_{i}, y_{i}\right), \quad i=1,2$. It comes up that

$$
\begin{aligned}
\mid \int_{\mathbb{R}^{2}}\left(h_{1, n} h_{2, n}\right)^{-1} \mathbb{K}\left(\frac{u_{1}}{h_{1, n}}, \frac{u_{2}}{h_{2, n}}\right) & f\left(u_{1}+y_{1}-\theta_{1} h_{1, n}, u_{2}+y_{2}-\theta_{2} h_{2, n}\right) d u_{1} d u_{2} \\
- & f\left(y_{1}, y_{2}\right) \int_{\mathbb{R}^{2}} \mathbb{K}\left(u_{1}, u_{2}\right) d u_{1} d u_{2} \mid \\
\leq & \mid \int_{\mathbb{R}^{2}}\left(h_{1, n} h_{2, n}\right)^{-1} \mathbb{K}\left(\frac{u_{1}}{h_{1, n}}, \frac{u_{2}}{h_{2, n}}\right) f\left(u_{1}+y_{1}, u_{2}+y_{2}\right) d u_{1} d u_{2} \\
& -\int_{\mathbb{R}^{2}}\left(h_{1, n} h_{2, n}\right)^{-1} \mathbb{K}\left(\frac{u_{1}}{h_{1, n}}, \frac{u_{2}}{h_{2, n}}\right) f\left(y_{1}, y_{2}\right) d u_{1} d u_{2} \mid .
\end{aligned}
$$

Let $g(x, y)$ an uniformly continuous function verifying

$$
\iint_{-\infty}^{+\infty}\left|g\left(v_{1}, v_{2}\right)\right| d v_{1} d v_{2}<\infty
$$

Let $h_{n}=\left(h_{1, n}, h_{2, n}\right)$ be a sequence of positive constants satisfing

$$
\lim _{n \rightarrow+\infty} h_{n}=0
$$

Define

$$
g_{n}\left(x_{1}, x_{2}\right)=\frac{1}{h_{1, n} h_{2, n}} \iint_{-\infty}^{+\infty} \mathbb{K}\left(\frac{v_{1}}{h_{1, n}}, \frac{v_{2}}{h_{2, n}}\right) g\left(x_{1}-v_{1}, x_{2}-v_{2}\right) d v_{1} d v_{2}
$$


Then

$$
\begin{aligned}
g_{n}\left(x_{1}, x_{2}\right)-g\left(x_{1}, x_{2}\right) \iint_{-\infty}^{+\infty} \mathbb{K}\left(v_{1}, v_{2}\right) d v_{1} d v_{2} \\
=\int_{\mathbb{R}^{2}}\left(h_{1, n} h_{2, n}\right)^{-1} \mathbb{K}\left(\frac{v_{1}}{h_{1, n}}, \frac{v_{2}}{h_{2, n}}\right)\left[g\left(x_{1}-v_{1}, x_{2}-v_{2}\right)-g\left(x_{1}, x_{2}\right)\right] d v_{1} d v_{2} .
\end{aligned}
$$

Let $\delta>0$ and split the region of integration into two regions: $\|v\| \leq \delta$ and $\|v\|>\delta$. Then we have

$$
\begin{aligned}
G & =\left|g_{n}\left(x_{1}, x_{2}\right)-g\left(x_{1}, x_{2}\right) \iint_{-\infty}^{+\infty} \mathbb{K}\left(v_{1}, v_{2}\right) d v_{1} d v_{2}\right| \\
& \leq \max _{\|v\| \leq \delta}\left|g\left(x_{1}-v_{1}, x_{2}-v_{2}\right)-g\left(x_{1}, x_{2}\right)\right| \iint_{\|z\| \leq \frac{\delta}{\|h\|}}\left|\mathbb{K}\left(z_{1}, z_{2}\right)\right| d z_{1} d z_{2} \\
& +\iint_{\|v\| \geq \delta} \frac{\left|g\left(x_{1}-v_{1}, x_{2}-v_{2}\right)\right|}{\|v\|} \frac{\|v\|}{h_{1, n} h_{2, n}} \mathbb{K}\left(\frac{v_{1}}{h_{1, n}}, \frac{v_{2}}{h_{2, n}}\right) d v_{1} d v_{2} \\
& +\left|g\left(x_{1}, x_{2}\right)\right| \iint_{\|v\| \geq \delta}\left(h_{1, n} h_{2, n}\right)^{-1} \mathbb{K}\left(\frac{u_{1}}{h_{1, n}}, \frac{u_{2}}{h_{2, n}}\right) d v_{1} d v_{2} \\
& \leq \max _{\|v\| \leq \delta}\left|g\left(x_{1}-v_{1}, x_{2}-v_{2}\right)-g\left(x_{1}, x_{2}\right)\right| \iint_{-\infty}^{+\infty}\left|\mathbb{K}\left(z_{1}, z_{2}\right)\right| d z_{1} d z_{2} \\
& +\frac{1}{\delta} \sup _{\|z\| \geq \frac{\delta}{h_{1, n} h_{2, n}}}\left|\left(z_{1}, z_{2}\right) \|\right| \mathbb{K}\left(z_{1}, z_{2}\right)\left|\iint_{\|z\| \geq \frac{\delta}{h_{1, n} h_{2, n}}}\right| g\left(z_{1}, z_{2}\right) \mid d z_{1} d z_{2} \\
& +\left|g\left(x_{1}, x_{2}\right)\right| \iint_{\|z\| \geq \frac{\delta}{h_{1, n} h_{2, n}}}\left|\mathbb{K}\left(z_{1}, z_{2}\right)\right| d z_{1} d z_{2} .
\end{aligned}
$$

As one lets $n$ tends to $\infty$, by choosing a small $\delta$, you may use the uniform continuity of $g$ to prove that the fisrt term tends to 0 . The second term tends to 0 , from the hypotheses on $\mathbb{K}(x, y)$. The third term too, since $\mathbb{K}(x, y)$ has compact support. Then at every point $\left(x_{1}, x_{2}\right)$, we have

$$
\lim _{n \rightarrow+\infty} g_{n}\left(x_{1}, x_{2}\right)=g\left(x_{1}, x_{2}\right) \iint_{-\infty}^{+\infty} \mathbb{K}\left(v_{1}, v_{2}\right) d v_{1} d v_{2}
$$

Therefore

$\lim _{n \rightarrow+\infty} \int_{\mathbb{R}^{2}}\left(h_{1, n} h_{2, n}\right)^{-1} \mathbb{K}\left(\frac{u_{1}}{h_{1, n}}, \frac{u_{2}}{h_{2, n}}\right) f\left(u_{1}+y_{1}, u_{2}+y_{2}\right) d u_{1} d u_{2}=f\left(y_{1}, y_{2}\right) \int_{\mathbb{R}^{2}} \mathbb{K}\left(u_{1}, u_{2}\right) d u_{1} d u_{2}$ uniformly, the proof of the lemma is then complete.

\subsection{Convergence of the estimator. Proof of the Lemma 1}

Observe first that

$$
\lim _{\left(z_{1}, z_{2}\right) \rightarrow(0,0)} \frac{F\left(z_{1}, z_{2}\right)-F\left(z_{1}, 0\right)-F\left(0, z_{2}\right)+F(0,0)}{z_{1} z_{2}}=f(0,0) .
$$


Indeed we have by the mean value theorem

$$
\left.F\left(z_{1}, z_{2}\right)-F\left(z_{1}, 0\right)=\left(z_{2}-0\right) \frac{\partial F}{\partial z_{2}}\left(z_{1}, s_{1}\right) \quad \text { with } \quad s_{1} \in\right] 0, z_{2}[,
$$

and

$$
\left.F(0,0)-F\left(0, z_{2}\right)=-\left(z_{2}-0\right) \frac{\partial F}{\partial z_{2}}\left(0, s_{1}\right) \quad \text { with } \quad s_{1} \in\right] 0, z_{2}[
$$

Therefore

$$
\begin{aligned}
F\left(z_{1}, z_{2}\right)-F\left(z_{1}, 0\right)-F\left(0, z_{2}\right)+F(0,0) & =z_{2}\left(\frac{\partial F}{\partial z_{2}}\left(z_{1}, s_{1}\right)-\frac{\partial F}{\partial z_{2}}\left(0, s_{1}\right)\right) \\
& \left.=z_{2}\left(z_{1}-0\right) \frac{\partial F}{\partial z_{1} \partial z_{2}}\left(s_{1}, s_{2}\right) \quad \text { with } \quad s_{1} \in\right] 0, z_{1}[ \\
& =z_{1} z_{2} \frac{\partial^{2} F}{\partial z_{1} \partial z_{2}}\left(s_{1}, s_{2}\right)
\end{aligned}
$$

Hence

$$
\frac{F\left(z_{1}, z_{2}\right)-F\left(z_{1}, 0\right)-F\left(0, z_{2}\right)+F(0,0)}{z_{1} z_{2}}=\frac{\partial^{2} F}{\partial z_{1} \partial z_{2}}\left(s_{1}, s_{2}\right)
$$

and

$$
\frac{\partial^{2} F}{\partial z_{1} \partial z_{2}}\left(s_{1}, s_{2}\right) \rightarrow \frac{\partial^{2} F}{\partial z_{1} \partial z_{2}}(0,0)=f(0,0) \quad \text { as } \quad\left(z_{1}, z_{2}\right) \rightarrow(0,0) .
$$

Therefore $\frac{\partial^{2} F}{z_{1} z_{2}}\left(z_{1}, z_{2}\right)$ is bounded.

Let $\quad \bar{\Delta}_{h_{1}, i}=\Delta_{h_{1}, i} \cap\left[0, z_{1}\right] ; \quad \bar{\Delta}_{h_{2}, j}=\Delta_{h_{2}, j} \cap\left[0, z_{2}\right] \quad$ and $\quad \chi_{B}$ the indicator function of the set $B=B_{1} \times B_{2}$. For $\left(z_{1}, z_{2}\right) \in\left[0, b_{1}\right] \times\left[0, b_{2}\right]$. We have

$$
\begin{aligned}
\mathbb{E}\left(P_{n}\left(z_{1}, z_{2}, \alpha_{1}, \alpha_{2}\right)\right)=\sum_{i=0}^{\left[\frac{z_{1}}{h_{1}}\right]} \sum_{j=0}^{\left[\frac{z_{2}}{h_{2}}\right]}(1 & \left.-\frac{i h_{1}}{z_{1}}\right)^{\alpha_{1}}\left(1-\frac{j h_{2}}{z_{2}}\right)^{\alpha_{2}} \int_{\mathbb{R}^{2}} \mathbb{K}\left(u_{1}, u_{2}\right) \\
& \times f\left(u_{1} h_{1}-i h_{1}, u_{2} h_{2}-j h_{2}\right) d u_{1} d u_{2}
\end{aligned}
$$

which can be written in the following form

$$
\begin{aligned}
\int_{0}^{z_{1}} \int_{0}^{z_{2}} \sum_{i=0}^{\left[\frac{z_{1}}{h_{1}}\right]} \chi_{\bar{\Delta}_{h_{1}, i}}(x) \sum_{j=0}^{\left[\frac{z_{2}}{h_{2}}\right]} \chi_{\bar{\Delta}_{h_{2}, j}}(y)\left(1-\frac{i h_{1}}{z_{1}}\right)^{\alpha_{1}}\left(1-\frac{j h_{2}}{z_{2}}\right)^{\alpha_{2}} \\
\times \iint_{-\infty}^{+\infty} \mathbb{K}\left(u_{1}, u_{2}\right) f\left(u_{1} h_{1}-i h_{1}, u_{2} h_{2}-j h_{2}\right) d u_{1} d u_{2} d x d y \\
+\left(h_{1}\left(\left[\frac{z_{1}}{h_{1}}\right]+1\right)-z_{1}\right)\left(h_{2}\left(\left[\frac{z_{2}}{h_{2}}\right]+1\right)-z_{2}\right)\left(1-\frac{h_{1}\left[\frac{z_{1}}{h_{1}}\right]}{z_{1}}\right)^{\alpha_{1}}\left(1-\frac{h_{2}\left[\frac{z_{2}}{h_{2}}\right]}{z_{2}}\right)^{\alpha_{2}} \\
\times \iint_{-\infty}^{+\infty} \mathbb{K}\left(u_{1}, u_{2}\right) f\left(u_{1} h_{1}+\frac{h_{1}\left[\frac{z_{1}}{h_{1}}\right]}{z_{1}}, u_{2} h_{2}-\frac{h_{2}\left[\frac{z_{2}}{h_{2}}\right]}{z_{2}}\right) d u_{1} d u_{2} .
\end{aligned}
$$


We have

$$
\begin{array}{r}
\sup _{\left(z_{1}, z_{2}\right) \in \mathbb{R}^{2}} \mid\left(h_{1}\left(\left[\frac{z_{1}}{h_{1}}\right]+1\right)-z_{1}\right)\left(h_{2}\left(\left[\frac{z_{2}}{h_{2}}\right]+1\right)-z_{2}\right)\left(1-\frac{h_{1}\left[\frac{z_{1}}{h_{1}}\right]}{z_{1}}\right)^{\alpha_{1}}\left(1-\frac{h_{2}\left[\frac{z_{2}}{h_{2}}\right]}{z_{2}}\right)^{\alpha_{2}} \\
\times \iint_{-\infty}^{+\infty} \mathbb{K}\left(u_{1}, u_{2}\right) f\left(u_{1} h_{1}+\frac{h_{1}\left[\frac{z_{1}}{h_{1}}\right]}{z_{1}}, u_{2} h_{2}-\frac{h_{2}\left[\frac{z_{2}}{h_{2}}\right]}{z_{2}}\right) d u_{1} d u_{2} \mid \\
\leq h_{1} h_{2} \sup _{(x, y) \in \mathbb{R}^{2}} f(x, y) \iint_{-\infty}^{+\infty}\left|\mathbb{K}\left(u_{1}, u_{2}\right)\right| d u_{1} d u_{2},
\end{array}
$$

since $\left|h_{i}\left(\left[\frac{z_{i}}{h_{i}}\right]+1\right)-z_{i}\right| \leq h_{i} \quad i=1,2$.

According to $\mathbf{H}_{\mathbf{2}}$, we can write

$$
P\left(z_{1}, z_{2}, \alpha_{1}, \alpha_{2}\right)=\iint_{-\infty}^{+\infty} \int_{0}^{z_{1}} \int_{0}^{z_{2}}\left(1-\frac{x}{z_{1}}\right)^{\alpha_{1}}\left(1-\frac{y}{z_{2}}\right)^{\alpha_{2}} \mathbb{K}\left(u_{1}, u_{2}\right) d u_{1} d u_{2} f(x, y) d x d y \text {. }
$$

Let $(x, y) \in \bar{\Delta}_{h_{1}, i} \times \bar{\Delta}_{h_{2}, j}$. By considering the terms in 4 and in 6 we get,

$$
\begin{aligned}
& \mid\left(1-\frac{i h_{1}}{z_{1}}\right)^{\alpha_{1}}\left(1-\frac{j h_{2}}{z_{2}}\right)^{\alpha_{2}} \iint_{-\infty}^{+\infty} \mathbb{K}\left(u_{1}, u_{2}\right) f\left(u_{1} h_{1}-i h_{1}, u_{1} h_{2}-j h_{2}\right) d u_{1} d u_{2} \\
& -\left(1-\frac{x}{z_{1}}\right)^{\alpha_{1}}\left(1-\frac{y}{z_{2}}\right)^{\alpha_{2}} \mathbb{K}\left(u_{1}, u_{2}\right) d u_{1} d u_{2} f(x, y) \mid \\
& =\mid \iint_{-\infty}^{+\infty}\left[\left(1-\frac{i h_{1}}{z_{1}}\right)^{\alpha_{1}}\left(1-\frac{j h_{2}}{z_{2}}\right)^{\alpha_{2}} f\left(u_{1} h_{1}-i h_{1}, u_{2} h_{2}-j h_{2}\right)\right. \\
& \left.-\left(1-\frac{x}{z_{1}}\right)^{\alpha_{1}}\left(1-\frac{y}{z_{2}}\right)^{\alpha_{2}} f(x, y)\right] \mathbb{K}\left(u_{1}, u_{2}\right) d u_{1} d u_{2} \mid \\
& \leq \iint_{-\infty}^{+\infty} \mid\left(1-\frac{i h_{1}}{z_{1}}\right)^{\alpha_{1}}\left(1-\frac{j h_{2}}{z_{2}}\right)^{\alpha_{2}}-\left(1-\frac{x}{z_{1}}\right)^{\alpha_{1}} \times \\
& \left(1-\frac{y}{z_{2}}\right)^{\alpha_{2}}|f(x, y)| \mathbb{K}\left(u_{1}, u_{2}\right) \mid d u_{1} d u_{2} \\
& +\iint_{-\infty}^{+\infty}\left|\left(1-\frac{i h_{1}}{z_{1}}\right)^{\alpha_{1}}\left(1-\frac{j h_{2}}{z_{2}}\right)^{\alpha_{2}}\right| \times \\
& \left|f\left(u_{1} h_{1}-i h_{1}, u_{1} h_{2}-j h_{2}\right)-f(x, y)\right|\left|\mathbb{K}\left(u_{1}, u_{2}\right)\right| d u_{1} d u_{2} .
\end{aligned}
$$

For $(x, y) \in \Delta_{h_{1}, i} \times \Delta_{h_{2}, j}$, we have by the first order Taylor formula applied to the function

$$
g(x, y)=\left(1-\frac{x}{z_{1}}\right)^{\alpha_{1}}\left(1-\frac{y}{z_{2}}\right)^{\alpha_{2}},
$$

for $\left.c_{1} \in\right] h_{1} i, x\left[\right.$ and $\left.c_{2} \in\right] h_{2} j, y[$,

$$
\begin{aligned}
& \left|\left(1-\frac{i h_{1}}{z_{1}}\right)^{\alpha_{1}}\left(1-\frac{j h_{2}}{z_{2}}\right)^{\alpha_{2}}-\left(1-\frac{x}{z_{1}}\right)^{\alpha_{1}}\left(1-\frac{y}{z_{2}}\right)^{\alpha_{2}}\right| \\
& =\mid\left(1-\frac{c_{1}}{z_{1}}\right)^{\alpha_{1}-1} \frac{\alpha_{1}}{z_{1}}\left(1-\frac{c_{2}}{z_{2}}\right)^{\alpha_{2}}\left(i h_{1}-x\right) \\
& +\left(1-\frac{c_{1}}{z_{1}}\right)^{\alpha_{1}} \frac{\alpha_{2}}{z_{2}}\left(1-\frac{c_{2}}{z_{2}}\right)^{\alpha_{2}-1}\left(j h_{2}-y\right) \mid \\
& \leq 2\left(\frac{\alpha_{1} h_{1}}{z_{1}}+\frac{\alpha_{2} h_{2}}{z_{2}}\right)=2\left(\frac{z_{2} \alpha_{1} h_{1}+z_{1} \alpha_{2} h_{2}}{z_{1} z_{2}}\right) .
\end{aligned}
$$


Therefore, denoting by $I_{1}^{i, j}(x, y)$ the first integral of the right hand-side of $(7)$ and

$$
I_{1}(x, y)=\sum_{i=0}^{\left[\frac{z_{1}}{h_{1}}\right]} \chi_{\bar{\Delta}_{h_{1}, i}}(x) \sum_{j=0}^{\left[\frac{z_{2}}{h_{2}}\right]} \chi_{\bar{\Delta}_{h_{2}, j}}(y) I_{1}^{i, j}(x, y)
$$

We have

$$
\begin{aligned}
\int_{0}^{z_{1}} \int_{0}^{z_{2}} I_{1}(x, y) d x d y & \leq \frac{2\left(\alpha_{1} z_{2}+\alpha_{2} z_{1}\right)\left\|\left(h_{1}, h_{2}\right)\right\|}{z_{1} z_{2}} \times \\
& \int_{0}^{z_{1}} \int_{0}^{z_{2}}\left(\iint_{-\infty}^{+\infty} f(x, y)\left|\mathbb{K}\left(u_{1}, u_{2}\right)\right| d u_{1} d u_{2}\right) d x d y \\
& =2\left(\alpha_{1} z_{2}+\alpha_{2} z_{1}\right)\left\|\left(h_{1}, h_{2}\right)\right\| \times \\
& \left(\iint_{-\infty}^{+\infty}\left|\mathbb{K}\left(u_{1}, u_{2}\right)\right| d u_{1} d u_{2}\right) \frac{F\left(z_{1}, z_{2}\right)}{z_{1} z_{2}} .
\end{aligned}
$$

Denoting by $I_{2}^{i, j}(x, y)$ the second integral of the right hand-side of $(7)$ and

$$
I_{2}(x, y)=\sum_{i=0}^{\left[\frac{z_{1}}{h_{1}}\right]} \chi_{\bar{\Delta}_{h_{1}, i}}(x) \sum_{j=0}^{\left[\frac{z_{2}}{h_{2}}\right]} \chi_{\bar{\Delta}_{h_{2}, j}}(y) I_{2}^{i, j}(x, y)
$$

We have

$$
\begin{aligned}
I_{2}^{i, j}(x, y) & \leq \iint_{-\infty}^{+\infty}\left|f\left(u_{1} h_{1}-i h_{1}, u_{2} h_{2}-j h_{2}\right)-f\left(i h_{1}, j h_{2}\right)\right|\left|\mathbb{K}\left(u_{1}, u_{2}\right)\right| d u_{1} d u_{2} \\
& +\iint_{-\infty}^{+\infty}\left|f\left(i h_{1}, j h_{2}\right)-f(x, y) \| \mathbb{K}\left(u_{1}, u_{2}\right)\right| d u_{1} d u_{2} .
\end{aligned}
$$

Let $\varepsilon>0$, since $f(x, y)$ is uniformly continuous, there exists $\eta_{0}=\eta_{0}\left(z_{1}, z_{2}\right)>0$ such that $\left\|\left(i h_{1}, j h_{2}\right)-(x, y)\right\|<\eta_{0}$, hence if $\|h\|<\eta_{0}$, we have $\mid f\left(u_{1} h_{1}-i h_{1}, u_{2} h_{2}-j h_{2}\right)-$ $f(x, y) \mid<\frac{\varepsilon}{\|b\|}$. Therefore

$$
\begin{aligned}
\int_{0}^{z_{1}} \int_{0}^{z_{2}} I_{2}(x, y) d x d y & \leq \sum_{i=0}^{\left[\frac{z_{1}}{h_{1}}\right]\left[\frac{z_{2}}{h_{2}}\right]} \sum_{j=0}^{+\infty} h_{1} h_{2} \iint_{-\infty}^{+\infty}\left|f\left(u_{1} h_{1}-i h_{1}, u_{2} h_{2}-j h_{2}\right)-f\left(i h_{1}, j h_{2}\right)\right| \\
& \times\left|\mathbb{K}\left(u_{1}, u_{2}\right)\right| d u_{1} d u_{2}+\varepsilon \iint_{-\infty}^{+\infty}\left|\mathbb{K}\left(u_{1}, u_{2}\right)\right| d u_{1} d u_{2} d x d y .
\end{aligned}
$$

By the uniform continuity of $f(x, y)$ we have

$$
\exists \quad \eta_{1}=\eta_{1}\left(z_{1}, z_{2}\right)>0,\|u h\|<\eta_{1} \Rightarrow\left|f\left(u_{1} h_{1}-i h_{1}, u_{2} h_{2}-j h_{2}\right)-f\left(i h_{1}, j h_{2}\right)\right|<\frac{\varepsilon}{\|b\|} .
$$


Hence

$$
\begin{aligned}
& \sum_{i=0}^{\left[\frac{z_{1}}{h_{1}}\right]\left[\frac{z_{2}}{h_{2}}\right]} \sum_{j=0} h_{1} h_{2} \iint_{-\infty}^{+\infty}\left|f\left(u_{1} h_{1}-i h_{1}, u_{2} h_{2}-j h_{2}\right)-f\left(i h_{1}, j h_{2}\right)\right|\left|\mathbb{K}\left(u_{1}, u_{2}\right)\right| d u_{1} d u_{2} \\
& \leq \sum_{i=0}^{\left[\frac{z_{1}}{h_{1}}\right]\left[\frac{z_{2}}{h_{2}}\right]} \sum_{j=0} h_{1} h_{2} \iint_{\|u h\|<\eta_{1}} \frac{\varepsilon}{\|b\|}\left|\mathbb{K}\left(u_{1}, u_{2}\right)\right| d u_{1} d u_{2} \\
& +\sum_{i=0} \sum_{j=0}^{\left[\frac{z_{1}}{h_{1}}\right]\left[\frac{z_{2}}{h_{2}}\right]} h_{1} h_{2} \iint_{\|u h\| \geq \eta_{1}}\left|f\left(u_{1} h_{1}-i h_{1}, u_{2} h_{2}-j h_{2}\right)-f\left(i h_{1}, j h_{2}\right)\right|\left|\mathbb{K}\left(u_{1}, u_{2}\right)\right| d u_{1} d u_{2} \\
& \leq \varepsilon \int_{-\infty}^{+\infty}\left|\mathbb{K}\left(u_{1}, u_{2}\right)\right| d u_{1} d u_{2}+\sum_{i=0}^{\left[\frac{z_{1}}{h_{1}}\right]\left[\frac{z_{2}}{h_{2}}\right]} \sum_{j=0} h_{1} h_{2} \iint_{\|u h\| \geq \eta_{1}}\left|f\left(u_{1} h_{1}-i h_{1}, u_{2} h_{2}-j h_{2}\right)\right| \\
& \quad \times\left|\mathbb{K}\left(u_{1}, u_{2}\right)\right| d u_{1} d u_{2}+\sum_{i=0}^{\left[\frac{z_{1}}{h_{1}}\right]\left[\frac{z_{2}}{h_{2}}\right]} \sum_{j=0}^{z_{1}} h_{1} h_{2} \iint_{\|u h\| \geq \eta_{1}}\left|f\left(i h_{1}, j h_{2}\right) \| \mathbb{K}\left(u_{1}, u_{2}\right)\right| d u_{1} d u_{2} .
\end{aligned}
$$

Since $f(x, y)$, is continuous, its is Riemann-integrable. Hence

$$
\sum_{i=0}^{\left[\frac{z_{1}}{h_{1}}\right]} \sum_{j=0}^{\left[\frac{z_{2}}{h_{2}}\right]} h_{1} h_{2}\left|f\left(i h_{1}, j h_{2}\right)\right| \rightarrow \int_{0}^{b_{1}} \int_{0}^{b_{2}} f(x, y) d x d y \quad n \rightarrow+\infty .
$$

Since

$$
\left(h_{1}\left(\left[\frac{b_{1}}{h_{1}}\right]+1\right)-b_{1}\right)\left(h_{2}\left(\left[\frac{b_{2}}{h_{2}}\right]+1\right)-b_{2}\right) f\left(h_{1}\left[\frac{b_{1}}{h_{1}}\right], h_{2}\left[\frac{b_{2}}{h_{2}}\right]\right) \rightarrow 0, \quad n \rightarrow+\infty,
$$

and

$$
\sum_{i=0}^{\left[\frac{z_{1}}{h_{1}}\right]} \sum_{j=0}^{\left[\frac{z_{2}}{h_{2}}\right]} h_{1} h_{2}\left|f\left(i h_{1}, j h_{2}\right)\right| \rightarrow \int_{0}^{b_{1}} \int_{0}^{b_{2}} f(x, y) d x d y .
$$

This last sum is bounded, let $A$ be its bound.

By the change of variables $v_{i}=u_{i} h_{i} \quad i=1,2$, we have

$$
\begin{gathered}
\sum_{i=0}^{\left[\frac{z_{1}}{h_{1}}\right]\left[\frac{z_{2}}{h_{2}}\right]} \sum_{j=0} h_{1} h_{2} \iint_{\|u h\| \geq \eta_{1}}\left|f\left(u_{1} h_{1}-i h_{1}, u_{2} h_{2}-j h_{2}\right)\right|\left|\mathbb{K}\left(u_{1}, u_{2}\right)\right| d u_{1} d u_{2} \\
\quad+\sum_{i=0}^{\left[\frac{z_{1}}{h_{1}}\right]\left[\frac{z_{2}}{h_{2}}\right]} \sum_{j=0} h_{1} h_{2} \iint_{\|u h\| \geq \eta_{1}}\left|f\left(i h_{1}, j h_{2}\right)\right|\left|\mathbb{K}\left(u_{1}, u_{2}\right)\right| d u_{1} d u_{2} \\
\leq \sum_{i=0}^{\left[\frac{z_{1}}{h_{1}}\right]\left[\frac{z_{2}}{h_{2}}\right]} \sum_{j=0} h_{1} h_{2} \iint_{\|v\| \geq \eta_{1}}\left|f\left(v_{1}-i h_{1}, v_{2}-j h_{2}\right)\right| \frac{1}{h_{1} h_{2}}\left|\mathbb{K}\left(\frac{v_{1}}{h_{1}}, \frac{v_{2}}{h_{2}}\right)\right| d v_{1} d v_{2} \\
+A \iint_{\|u h\| \geq \eta_{1}}\left|\mathbb{K}\left(u_{1}, u_{2}\right)\right| d u_{1} d u_{2} .
\end{gathered}
$$


According to $\left(\mathbf{H}_{\mathbf{3}}\right)$ there exists $C>0$ such that for $\left|\frac{v_{1}}{h_{1}} \frac{v_{2}}{h_{2}}\right| \geq C$, we have

$$
\left|\frac{v_{1}}{h_{1}} \frac{v_{2}}{h_{2}} \| \mathbb{K}\left(\frac{v_{1}}{h_{1}}, \frac{v_{2}}{h_{2}}\right)\right| \leq \frac{\eta_{1} \varepsilon}{\|b\|} .
$$

Let $\eta=\inf \left(\eta_{1}, C h_{1} h_{2}\right), \quad i=1,2$ being small enough, then

$$
\begin{aligned}
& \frac{1}{\eta_{1}} \iint_{\|v\| \geq \eta_{1}}\left|f\left(v_{1}-i h_{1}, v_{2}-j h_{2}\right) \frac{v_{1}}{h_{1}} \frac{v_{2}}{h_{2}} \mathbb{K}\left(\frac{v_{1}}{h_{1}}, \frac{v_{2}}{h_{2}}\right)\right| d v_{1} d v_{2} \\
& \leq \frac{\varepsilon}{\|b\|} \iint_{\|v\| \geq \eta_{1}}\left|f\left(v_{1}-i h_{1}, v_{2}-j h_{2}\right)\right| d v_{1} d v_{2} \\
& \leq \frac{\varepsilon}{\|b\|} \iint_{\mathbb{R}^{2}}|f(x, y)| d x d y=\frac{\varepsilon}{\|b\|}
\end{aligned}
$$

Where

$$
\sum_{i=0}^{\left[\frac{z_{1}}{h_{1}}\right]\left[\frac{z_{2}}{h_{2}}\right]} \sum_{j=0} h_{1} h_{2} \iint_{\|v\| \geq \eta_{1}}\left|f\left(v_{1}-i h_{1}, v_{2}-j h_{2}\right)\right| \frac{1}{h_{1} h_{2}}\left|\mathbb{K}\left(\frac{v_{1}}{h_{1}}, \frac{v_{2}}{h_{2}}\right)\right| d v_{1} d v_{2} \leq \frac{z_{1} z_{2} \varepsilon}{\|b\|} \leq \varepsilon
$$

Since $A \iint_{\|u h\| \geq \eta_{1}}\left|\mathbb{K}\left(u_{1}, u_{2}\right)\right| d u_{1} d u_{2} \rightarrow 0, n \rightarrow+\infty$, we have together with 8 and 5

$$
\begin{aligned}
\lim _{n \rightarrow+\infty} \sup _{\left(z_{1}, z_{2}\right) \in\left[0, b_{1}\right] \times\left[0, b_{2}\right]}\left|\mathbb{E}\left(P_{n}\left(z_{1}, z_{2}, \alpha_{1}, \alpha_{2}\right)\right)-P\left(z_{1}, z_{2}, \alpha_{1}, \alpha_{2}\right)\right| & \\
& \leq 2 \varepsilon \iint_{-\infty}^{+\infty} \mathbb{K}\left(u_{1}, u_{2}\right) d u_{1} d u_{2}+\varepsilon
\end{aligned}
$$

The proof of the lemma is complete.

\subsection{Proof of Lemma 2}

For $(x, y) \in \bar{\Delta}_{h_{1}, i} \times \bar{\Delta}_{h_{2}, j} \quad i=1, \ldots,\left[\frac{z_{1}}{h_{1}}\right] ; j=1, \ldots,\left[\frac{z_{2}}{h_{2}}\right]$, we have

$$
\begin{gathered}
\iint_{-\infty}^{+\infty}\left|\left(1-\frac{i h_{1}}{z_{1}}\right)^{\alpha_{1}}\left(1-\frac{j h_{2}}{z_{2}}\right)^{\alpha_{2}}\right|\left|f\left(u_{1} h_{1}-i h_{1}, u_{1} h_{2}-j h_{2}\right)\right|\left|\mathbb{K}\left(u_{1}, u_{2}\right)\right| d u_{1} d u_{2} \\
\leq\left(\iint_{-\infty}^{+\infty} \int_{x}^{x+h_{1}\left(\left|u_{1}\right|+1\right)} \int_{y}^{y+h_{2}\left(\left|u_{2}\right|+1\right)}\left|f^{\prime}\left(t_{1}, t_{2}\right)\right|\left|\mathbb{K}\left(u_{1}, u_{2}\right)\right| d u_{1} d u_{2}\right) .
\end{gathered}
$$

Hence $I_{2}(x, y)$ being as in the previous Lemma 1, the following inequality holds

$$
\begin{aligned}
& \int_{0}^{z_{1}} \int_{0}^{z_{2}} I_{2}(x, y) d x d y \\
& \leq \int_{0}^{z_{1}} \int_{0}^{z_{2}}\left(\iint_{-\infty}^{+\infty} \int_{x}^{x+h_{1}\left(\left|u_{1}\right|+1\right)} \int_{y}^{y+h_{2}\left(\left|u_{2}\right|+1\right)}\left|f^{\prime}\left(t_{1}, t_{2}\right)\right|\left|\mathbb{K}\left(u_{1}, u_{2}\right)\right| d u_{1} d u_{2}\right) d x d y
\end{aligned}
$$


By the change of variables $t_{1}=x+h_{1}\left(\left|u_{1}\right|+1\right) v_{1} ; t_{2}=y+h_{2}\left(\left|u_{2}\right|+1\right) v_{2}$, we have by Fubini's theorem

$$
\begin{aligned}
& \int_{0}^{z_{1}} \int_{0}^{z_{2}} I_{2}(x, y) d x d y \leq h_{1} h_{2} \iint_{\mathbb{R}^{2}}\left(\left|u_{1}\right|+1\right)\left(\left|u_{2}\right|+1\right)\left|\mathbb{K}\left(u_{1}, u_{2}\right)\right| \\
& \times\left(\iint_{\mathbb{R}^{2}}\right)\left|f^{\prime}\left(x+h_{1}\left(\left|u_{1}\right|+1\right) v_{1}, y+h_{2}\left(\left|u_{2}\right|+1\right) v_{2}\right)\right| d x d y d u_{1} d u_{2} \iint_{0}^{1} d v_{1} d v_{2} \\
& \quad=\iint_{\mathbb{R}^{2}}\left|f^{\prime}(x, y)\right| d x d y .
\end{aligned}
$$

This inequality together with inequality 5 and 8 lead to the completion of the proof.

\subsection{Proof of Theorem 1 and Theorem 2}

Let $\hat{F}_{n}$ be the empirical distribution of the sample $\left(X_{1}, Y_{1}\right),\left(X_{2}, Y_{2}\right), \ldots,\left(X_{n}, Y_{n}\right)$ define by

$$
F_{n}\left(l_{1}, l_{2}\right)=n^{-1} \sum_{i=1}^{n} \sum_{j=1}^{m}\left(\chi_{X_{i}<l_{1}} \chi_{Y_{j}<l_{2}}\right)
$$

where $\chi_{A}$ stands for the indicator function of a set $A$. We can write

$P_{n}\left(z_{1}, z_{2}, \alpha_{1}, \alpha_{2}\right)=\iint_{\mathbb{R}^{2}} \sum_{i=0}^{\left[\frac{z_{1}}{h_{1}}\right]\left[\frac{z_{2}}{h_{2}}\right]} \sum_{j=0}\left(1-\frac{i h_{1}}{z_{1}}\right)^{\alpha_{1}}\left(1-\frac{j h_{2}}{z_{2}}\right)^{\alpha_{2}} \mathbb{K}\left(\frac{l_{1}-i h_{1}}{h_{1}}, \frac{l_{2}-j h_{2}}{h_{2}}\right) d \hat{F}_{n}\left(l_{1}, l_{2}\right)$,

and

$\mathbb{E}\left(P_{n}\left(z_{1}, z_{2}, \alpha_{1}, \alpha_{2}\right)\right)=\sum_{i=0}^{\left[\frac{z_{1}}{h_{1}}\right]} \sum_{j=0}^{\left[\frac{z_{2}}{h_{2}}\right]} \iint_{\mathbb{R}^{2}}\left(1-\frac{i h_{1}}{z_{1}}\right)^{\alpha_{1}}\left(1-\frac{j h_{2}}{z_{2}}\right)^{\alpha_{2}} \mathbb{K}\left(\frac{l_{1}-i h_{1}}{h_{1}}, \frac{l_{2}-j h_{2}}{h_{2}}\right) d F\left(l_{1}, l_{2}\right)$.

We have

$$
\begin{aligned}
& \left|P_{n}\left(z_{1}, z_{2}, \alpha_{1}, \alpha_{2}\right)-\mathbb{E}\left(P_{n}\left(z_{1}, z_{2}, \alpha_{1}, \alpha_{2}\right)\right)\right| \\
& =\left|\sum_{i=0}^{\left[\frac{z_{1}}{h_{1}}\right]} \sum_{j=0}^{\left[\frac{z_{2}}{h_{2}}\right]} \iint_{\mathbb{R}^{2}}\left(1-\frac{i h_{1}}{z_{1}}\right)^{\alpha_{1}}\left(1-\frac{j h_{2}}{z_{2}}\right)^{\alpha_{2}} \mathbb{K}\left(\frac{l_{1}-i h_{1}}{h_{1}}, \frac{l_{2}-j h_{2}}{h_{2}}\right)\left(d \hat{F}_{n}\left(l_{1}, l_{2}\right)-d F\left(l_{1}, l_{2}\right)\right)\right| .
\end{aligned}
$$

First we are going to apply the Fubini's theorem and then integrate by parts with respect to for each variable. 
We obtain then

$$
\begin{aligned}
& \iint_{\mathbb{R}^{2}} \mathbb{K}\left(\frac{l_{1}-i h_{1}}{h_{1}}\right.\left., \frac{l_{2}-j h_{2}}{h_{2}}\right)\left(d \hat{F}_{n}\left(l_{1}, l_{2}\right)-d F\left(l_{1}, l_{2}\right)\right) \\
&=\int_{\mathbb{R}} K\left(\frac{l_{1}-i h_{1}}{h_{1}}\right) \int_{\mathbb{R}} K\left(\frac{l_{2}-j h_{2}}{h_{2}}\right)\left(d \hat{F}_{n}\left(l_{1}, l_{2}\right)-d F\left(l_{1}, l_{2}\right)\right) \\
&=\int_{\mathbb{R}} K\left(\frac{l_{1}-i h_{1}}{h_{1}}\right)\left[\int_{\mathbb{R}} K\left(\frac{l_{2}-j h_{2}}{h_{2}}\right) \frac{\partial^{2}\left(\hat{F}_{n}\left(l_{1}, l_{2}\right)-F\left(l_{1}, l_{2}\right)\right)}{\partial l_{1} \partial l_{2}} d l_{1} d l_{2}\right] \\
&=\int_{\mathbb{R}} K\left(\frac{l_{1}-i h_{1}}{h_{1}}\right)\left\{\left[K\left(\frac{l_{2}-j h_{2}}{h_{2}}\right) \frac{\partial\left(\hat{F}_{n}\left(l_{1}, l_{2}\right)-F\left(l_{1}, l_{2}\right)\right)}{\partial l_{1}} d l_{1}\right]_{-\infty}^{+\infty}\right. \\
&=-\int_{\mathbb{R}} K\left(\frac{l_{1}-i h_{1}}{h_{1}}\right) \frac{\partial\left(\hat{F}_{n}\left(l_{1}, l_{2}\right)-F\left(l_{1}, l_{2}\right)\right)}{\partial l_{1}} d l_{1} \int_{\mathbb{R}} \frac{\partial K\left(\frac{l_{2}-j h_{2}}{h_{2}}\right)}{\partial l_{2}} d l_{2} \partial\left(\hat{F}_{n}\left(l_{1}, l_{2}\right)-F\left(l_{1}, l_{2}\right)\right) \\
&=-\left\{\left[l_{1}\right.\right.\left.\left.K\left(\frac{l_{1}-i h_{1}}{h_{1}}\right)\left(\hat{F}_{n}\left(l_{1}, l_{2}\right)-F\left(l_{1}, l_{2}\right)\right)\right]_{-\infty}^{+\infty}-\int_{\mathbb{R}} \frac{\partial K\left(\frac{l_{1}-i h_{1}}{h_{1}}\right)}{\partial l_{1}} d l_{1}\left(\hat{F}_{n}\left(l_{1}, l_{2}\right)-F\left(l_{1}, l_{2}\right)\right)\right\} \\
& \quad \times \int_{\mathbb{R}} \frac{\partial K\left(\frac{l_{2}-j h_{2}}{h_{2}}\right)}{\partial l_{2}} d l_{2} \\
&=\int_{\mathbb{R}^{2}} d K\left(\frac{l_{1}-i h_{1}}{h_{1}}\right) d K\left(\frac{l_{2}-i h_{2}}{h_{2}}\right)\left(\hat{F}_{n}\left(l_{1}, l_{2}\right)-F\left(l_{1}, l_{2}\right)\right),
\end{aligned}
$$

where $d_{l_{i}} K(\cdot)=\frac{\partial K(\cdot) d l_{i}}{\partial l_{i}}$ denote the derivative of $K$ with respect to $l_{i}$. Therefore

$$
\begin{aligned}
& \left|P_{n}\left(z_{1}, z_{2}, \alpha_{1}, \alpha_{2}\right)-\mathbb{E}\left(P_{n}\left(z_{1}, z_{2}, \alpha_{1}, \alpha_{2}\right)\right)\right| \\
& \leq \sum_{i=0}^{\left[\frac{z_{1}}{h_{1}}\right]\left[\frac{z_{2}}{h_{2}}\right]} \sum_{j=0} \iint_{\mathbb{R}^{2}}\left|d K\left(\frac{l_{1}-i h_{1}}{h_{1}}\right) d K\left(\frac{l_{2}-i h_{2}}{h_{2}}\right)\right| \sup _{\left(l_{1}, l_{2}\right) \in \mathbb{R}^{2}}\left|\hat{F}_{n}\left(l_{1}, l_{2}\right)-F\left(l_{1}, l_{2}\right)\right| \\
& \leq \sum_{i=0}^{\left[\frac{z_{1}}{h_{1}}\right]} \sum_{j=0}^{\left[\frac{z_{2}}{h_{2}}\right]} \int_{\mathbb{R}} d V_{-\infty}^{\frac{l_{1}-i h_{1}}{h_{1}}} K \int_{\mathbb{R}} d V_{-\infty}^{\frac{l_{2}-i h_{2}}{h_{2}}} \sup _{\left(l_{1}, l_{2}\right) \in \mathbb{R}^{2}}\left|\hat{F}_{n}\left(l_{1}, l_{2}\right)-F\left(l_{1}, l_{2}\right)\right| \\
& \leq\left[\frac{z_{1}}{h_{1}}\right]\left[\frac{z_{2}}{h_{2}}\right] V(\mathbb{R}) \times V(\mathbb{R}) \sup _{\left(l_{1}, l_{2}\right) \in \mathbb{R}^{2}}\left|\hat{F}_{n}\left(l_{1}, l_{2}\right)-F\left(l_{1}, l_{2}\right)\right| .
\end{aligned}
$$

Remarking that

$$
\begin{aligned}
& \left.\mid P_{n}\left(z_{1}, z_{2}, \alpha_{1}, \alpha_{2}\right)-\left(P_{(} z_{1}, z_{2}, \alpha_{1}, \alpha_{2}\right)\right) \mid \\
& \quad=\left|P_{n}\left(z_{1}, z_{2}, \alpha_{1}, \alpha_{2}\right)-\mathbb{E}\left(P_{n}\left(z_{1}, z_{2}, \alpha_{1}, \alpha_{2}\right)\right)+\mathbb{E}\left(P_{n}\left(z_{1}, z_{2}, \alpha_{1}, \alpha_{2}\right)\right)-P\left(z_{1}, z_{2}, \alpha_{1}, \alpha_{2}\right)\right| \\
& \quad \leq\left|P_{n}\left(z_{1}, z_{2}, \alpha_{1}, \alpha_{2}\right)-\mathbb{E}\left(P_{n}\left(z_{1}, z_{2}, \alpha_{1}, \alpha_{2}\right)\right)\right|+\left|\mathbb{E}\left(P_{n}\left(z_{1}, z_{2}, \alpha_{1}, \alpha_{2}\right)\right)-P\left(z_{1}, z_{2}, \alpha_{1}, \alpha_{2}\right)\right| .
\end{aligned}
$$

By the Lemma 1 we have

$$
\left|\mathbb{E}\left(P_{n}\left(z_{1}, z_{2}, \alpha_{1}, \alpha_{2}\right)\right)-P\left(z_{1}, z_{2}, \alpha_{1}, \alpha_{2}\right)\right| \rightarrow 0 \quad n \rightarrow+\infty,
$$


and because of the previous inequality we have

$$
\left|P_{n}\left(z_{1}, z_{2}, \alpha_{1}, \alpha_{2}\right)-\mathbb{E}\left(P_{n}\left(z_{1}, z_{2}, \alpha_{1}, \alpha_{2}\right)\right)\right| \rightarrow 0 \quad n \rightarrow+\infty
$$

\subsection{Proof of Corollary 2}

We have

$$
\begin{aligned}
& \left|P_{n}\left(z_{1}, z_{2}, \alpha_{1}, \alpha_{2}\right)-P\left(z_{1}, z_{2}, \alpha_{1}, \alpha_{2}\right)\right| \\
& =\left|\mathbb{E}\left(P_{n}\left(z_{1}, z_{2}, \alpha_{1}, \alpha_{2}\right)\right)-P\left(z_{1}, z_{2}, \alpha_{1}, \alpha_{2}\right)+P_{n}\left(z_{1}, z_{2}, \alpha_{1}, \alpha_{2}\right)-\mathbb{E}\left(P_{n}\left(z_{1}, z_{2}, \alpha_{1}, \alpha_{2}\right)\right)\right| .
\end{aligned}
$$

One other hand we have

$$
\begin{aligned}
& \left|P_{n}\left(z_{1}, z_{2}, \alpha_{1}, \alpha_{2}\right)-P\left(z_{1}, z_{2}, \alpha_{1}, \alpha_{2}\right)\right| \\
& \leq\left|\mathbb{E}\left(P_{n}\left(z_{1}, z_{2}, \alpha_{1}, \alpha_{2}\right)\right)-P\left(z_{1}, z_{2}, \alpha_{1}, \alpha_{2}\right)\right|+\left|P_{n}\left(z_{1}, z_{2}, \alpha_{1}, \alpha_{2}\right)-\mathbb{E}\left(P_{n}\left(z_{1}, z_{2}, \alpha_{1}, \alpha_{2}\right)\right)\right| .
\end{aligned}
$$

On the other hand by the Lemma 2 we have

$$
\begin{aligned}
& \sup _{\left(z_{1}, z_{2}\right) \in \mathbb{R}^{2}}\left|\mathbb{E}\left(P_{n}\left(z_{1}, z_{2}, \alpha_{1}, \alpha_{2}\right)\right)-P\left(z_{1}, z_{2}, \alpha_{1}, \alpha_{2}\right)\right| \leq \\
& h_{1} h_{2}\left(\left(\int_{\mathbb{R}^{2}}\left|f^{\prime}(x, y)\right| d x d y\right)\left(\int_{\mathbb{R}^{2}}(|u|+1)(|v|+1)|\mathbb{K}(u, v)| d u d v\right)\right. \\
& \left.+4\left(\alpha_{1} \alpha_{2} M+A h_{1} h_{2}\right) \int_{-\infty}^{+\infty}|\mathbb{K}(u, v)| d u d v\right),
\end{aligned}
$$

and moreover

$$
\left|P_{n}\left(z_{1}, z_{2}, \alpha_{1}, \alpha_{2}\right)-\mathbb{E}\left(P_{n}\left(z_{1}, z_{2}, \alpha_{1}, \alpha_{2}\right)\right)\right| \leq\left[\frac{z_{1}}{h_{1}}\right]\left[\frac{z_{2}}{h_{2}}\right] V(\mathbb{R}) \times V(\mathbb{R}) \sup _{\left(l_{1}, l_{2}\right) \in \mathbb{R}^{2}}\left|\hat{F}_{n}\left(l_{1}, l_{2}\right)-F\left(l_{1}, l_{2}\right)\right| .
$$

Therefore the second term of the right hand-side of inequality 9 tends to zero as $n \rightarrow$ $+\infty$. The first term tends to $h_{1} h_{2}=O\left(n^{-1} \log \log n\right)^{1 / 4}$ as $n \rightarrow+\infty$.

Hence the proof is complete.

\subsection{The uniform mean square consistency. Proof of Lemma 3}

Let $\delta=\left(\delta_{1}, \delta_{2}\right)>0$, and $\theta_{1}^{i}, \theta_{2}^{i} \quad i=1,2 ; i$ represent indices not powers. 
Define

$$
\begin{aligned}
& I_{n}\left(x_{1}, x_{2}, y_{1}, y_{2}\right)=\left(h_{1} h_{2}\right)^{-2} \iint_{-\infty}^{+\infty} \mid \mathbb{K}\left(\frac{u_{1}-x_{1}+\theta_{1}^{1} h_{1}}{h_{1}}, \frac{u_{2}-x_{2}+\theta_{1}^{2} h_{2}}{h_{2}}\right) \\
& \times \mathbb{K}\left(\frac{u_{1}-y_{1}+\theta_{2}^{1} h_{1}}{h_{1}}, \frac{u_{2}-y_{2}+\theta_{2}^{2} h_{2}}{h_{2}}\right) \mid f\left(u_{1}, u_{2}\right) d u_{1} d u_{2} \\
& =\iint_{-\infty}^{+\infty}\left(\left(h_{1} h_{2}\right)^{-1} \mathbb{K}\left(\frac{v_{1}}{h_{1}}, \frac{v_{2}}{h_{2}}\right)\right) \mid\left(\left(h_{1} h_{2}\right)^{-1} K\left(\frac{v_{1}+x_{1}-\theta_{1}^{1}-y_{1}+\theta_{2}^{1} h_{1}}{h_{1}}\right)\right. \\
& \left.\times K\left(\frac{v_{2}+x_{2}-\theta_{1}^{2}-y_{2}+\theta_{2}^{2} h_{2}}{h_{2}}\right)\right) \mid f\left(x_{1}+v_{1}-\theta_{1}^{1} h_{1}, x_{2}+v_{2}-\theta_{1}^{2} h_{2}\right) d u_{1} d u_{2} \\
& =\int_{\left|v_{1}-\theta_{1}^{1} h_{1}\right| \leq \delta_{1}} \int_{\left|v_{2}-\theta_{1}^{2} h_{2}\right| \leq \delta_{2}}+\int_{\left|v_{1}-\theta_{1}^{1} h_{1}\right|>\delta_{1}} \int_{\left|v_{2}-\theta_{1}^{2} h_{2}\right|>\delta_{2}} \int_{\left|v_{2}-\theta_{1}^{2} h_{2}\right|>\delta_{2}}+\int_{\left|v_{1}-\theta_{1}^{1} h_{1}\right|>\delta_{1}} \int_{\left|v_{2}-\theta_{1}^{2} h_{2}\right| \leq \delta_{2}} .
\end{aligned}
$$

Since $f$ is continuous, it is bounded on $I_{1} \times I_{2}=\left[x_{1}-\delta_{1}, x_{1}+\delta_{1}\right] \times\left[x_{2}-\delta_{2}, x_{2}+\delta_{2}\right]$. We assume that $n$ is large enough such that $\left(x_{1}+v_{1} \pm \theta_{1}^{1} h_{1}, x_{2}+v_{2} \pm \theta_{1}^{2} h_{2}\right) \in I_{1} \times I_{2}$. Therefore

$$
\begin{aligned}
& \int_{\left|v_{1}-\theta_{1}^{1} h_{1}\right| \leq \delta_{1}} \int_{\left|v_{1}-\theta_{1}^{1} h_{1}\right| \leq \delta_{2}} \leq \sup _{\left|v_{1}-\theta_{1}^{1} h_{1}\right| \leq \delta_{1}} \sup _{\left|v_{2}-\theta_{1}^{2} h_{2}\right| \leq \delta_{2}} f\left(x_{1}+v_{1}-\theta_{1}^{1} h_{1}, x_{2}+v_{2}-\theta_{1}^{2} h_{2}\right) \\
& \times \int_{-\frac{\delta_{1}}{h_{1}}+\theta_{1}^{1} \leq u_{1} \leq \frac{\delta_{1}}{h_{1}}+\theta_{1}^{1}} \int_{-\frac{\delta_{1}}{h_{1}}+\theta_{1}^{1} \leq u_{1} \leq \frac{\delta_{1}}{h_{1}}+\theta_{1}^{1}}\left|\mathbb{K}\left(u_{1}, u_{2}\right)\right| \\
& \times\left|\mathbb{K}\left(\frac{x_{1}-\theta_{1}^{1} h_{1}-y_{1}+\theta_{2}^{1} h_{1}}{h_{1}}+u_{1}, \frac{x_{2}-\theta_{1}^{2} h_{2}-y_{2}+\theta_{2}^{2} h_{2}}{h_{2}}+u_{2}\right)\left(h_{1} h_{2}\right)^{-1}\right| d u_{1} d u_{2} \\
& =\sup _{\left|v_{1}-\theta_{1}^{1} h_{1}\right| \leq \delta_{1}\left|v_{2}-\theta_{1}^{2} h_{2}\right| \leq \delta_{2}} f\left(x_{1}+v_{1}-\theta_{1}^{1} h_{1}, x_{2}+v_{2}-\theta_{1}^{2} h_{2}\right) \\
& \times \iint_{-\infty}^{+\infty} \chi_{-\frac{\delta_{1}}{h_{1}}+\theta_{1}^{1} \leq u_{1} \leq \delta_{1}}^{h_{1}}+\theta_{1}^{1}\left(u_{1}\right) \chi_{-\frac{\delta_{2}}{h_{2}}+\theta_{1}^{2} \leq u_{2} \leq \delta_{2}}+\theta_{1}^{2}\left(u_{2}\right)\left|\mathbb{K}\left(u_{1}, u_{2}\right)\right| \\
& \quad \times\left|\mathbb{K}\left(\frac{x_{1}-\theta_{1}^{1} h_{1}-y_{1}+\theta_{2}^{1} h_{1}}{h_{1}}+u_{1}, \frac{x_{2}-\theta_{1}^{2} h_{2}-y_{2}+\theta_{2}^{2} h_{2}}{h_{2}}+u_{2}\right)\left(h_{1} h_{2}\right)^{-1}\right| d u_{1} d u_{2} .
\end{aligned}
$$

For all $\left(u_{1}, u_{2}\right)$,

$$
\lim _{n \rightarrow+\infty}\left|\mathbb{K}\left(\frac{x_{1}-\theta_{1}^{1} h_{1}-y_{1}+\theta_{2}^{1} h_{1}}{h_{1}}+u_{1}, \frac{x_{2}-\theta_{1}^{2} h_{2}-y_{2}+\theta_{2}^{2} h_{2}}{h_{2}}+u_{2}\right)\left(h_{1} h_{2}\right)^{-1}\right|=0 .
$$

Write

$$
\begin{aligned}
& \left|\mathbb{K}\left(\frac{x_{1}-\theta_{1}^{1} h_{1}-y_{1}+\theta_{2}^{1} h_{1}}{h_{1}}+u_{1}, \frac{x_{2}-\theta_{1}^{2} h_{2}-y_{2}+\theta_{2}^{2} h_{2}}{h_{2}}+u_{2}\right)\left(h_{1} h_{2}\right)^{-1}\right| \\
& =\mid\left(\frac{x_{1}-\theta_{1}^{1} h_{1}-y_{1}+\theta_{2}^{1} h_{1}}{h_{1}}+u_{1}\right)\left(\frac{x_{2}-\theta_{1}^{2} h_{2}-y_{2}+\theta_{2}^{2} h_{2}}{h_{2}}+u_{2}\right) K\left(\frac{x_{1}-\theta_{1}^{1}-y_{1}+\theta_{2}^{1} h_{1}}{h_{1}}+u_{1}\right) \\
& \times K\left(\frac{x_{2}-\theta_{1}^{2}-y_{2}+\theta_{2}^{2} h_{2}}{h_{2}}+u_{2}\right) \mid \\
& \times\left|\frac{1}{x_{1}-\theta_{1}^{1} h_{1}-y_{1}+\theta_{2}^{1} h_{1}+h_{1} u_{1}} \times \frac{1}{x_{2}-\theta_{1}^{2} h_{2}-y_{2}+\theta_{2}^{2} h_{2}+h_{2} u_{2}}\right| .
\end{aligned}
$$


We have

$$
\left|\frac{1}{x_{i}-\theta_{1}^{i} h_{i}-y_{i}+\theta_{2}^{i} h_{i}+h_{i} u_{i}}\right|=\frac{1}{\left|x_{i}-y_{i}\right|\left|1-\frac{\theta_{1}^{i}-\theta_{2}^{i}-u_{i}}{x_{i}-y_{i}} h_{i}\right|} \quad i=1,2 .
$$

Since $\left|u_{i}\right| \leq \frac{\delta_{i}}{h_{i}}+\theta_{1}^{i}$, we may choose $\delta_{i}$ small enough such that for $n \geq n_{0}$, we have

$$
\left|\frac{\theta_{1}^{i}-\theta_{2}^{i}-u_{i}}{x_{i}-y_{i}} h_{i}\right| \leq \frac{3 h_{i}+\delta_{i}}{\left|x_{i}-y_{i}\right|}=\eta_{i}<1
$$

Therefore

$$
\left|\frac{1}{x_{i}-\theta_{1}^{i} h_{i}-y_{i}+\theta_{2}^{i} h_{i}+h_{i} u_{i}}\right| \leq \frac{1}{\left|x_{i}-y_{i}\right|\left(1-\eta_{i}\right)} .
$$

Since $\mathbf{H}_{\mathbf{3}}$ implies there exists $B$ such that

$$
\begin{aligned}
& \mid\left(\frac{x_{1}-\theta_{1}^{1} h_{1}-y_{1}+\theta_{2}^{1} h_{1}}{h_{1}}+u_{1}\right)\left(\frac{x_{2}-\theta_{1}^{2} h_{2}-y_{2}+\theta_{2}^{2} h_{2}}{h_{2}}+u_{2}\right) \\
& \times K\left(\frac{x_{1}-\theta_{1}^{1} h_{1}-y_{1}+\theta_{2}^{1} h_{1}}{h_{1}}+u_{1}\right) K\left(\frac{x_{2}-\theta_{1}^{2} h_{2}-y_{2}+\theta_{2}^{2} h_{2}}{h_{2}}+u_{2}\right) \mid \leq B, \\
& \left|\mathbb{K}\left(\frac{x_{1}-\theta_{1}^{1} h_{1}-y_{1}+\theta_{2}^{1} h_{1}}{h_{1}}+u_{1}, \frac{x_{2}-\theta_{1}^{2} h_{2}-y_{2}+\theta_{2}^{2} h_{2}}{h_{2}}+u_{2}\right)\left(h_{1} h_{2}\right)^{-1}\right| \\
& \leq \frac{2 B}{\left|x_{1}-y_{1}\right|\left(1-\eta_{1}\right)\left|x_{2}-y_{2}\right|\left(1-\eta_{2}\right)} .
\end{aligned}
$$

$\left|\mathbb{K}\left(u_{1}, u_{2}\right)\right|$ being integrable, by dominated convergence we get,

$$
\int_{\left|v_{1}-\theta_{1}^{1} h_{1}\right| \leq \delta_{1}} \int_{\left|v_{1}-\theta_{1}^{1} h_{1}\right| \leq \delta_{2}} \rightarrow 0 \quad \text { as } n \rightarrow+\infty .
$$

Let $\int_{\left|v_{1}-\theta_{1}^{1} h_{1}\right|>\delta_{1}} \int_{\left|v_{2}-\theta_{1} 2 h_{2}\right|>\delta_{2}}$ write it in the form

$$
\begin{aligned}
\int_{\left|v_{1}-\theta_{1}^{1} h_{1}\right|>\delta_{1}} \int_{\left|v_{2}-\theta_{1}^{2} h_{2}\right|>\delta_{2}} & =\int_{\left|v_{1}-\theta_{1}^{1} h_{1}\right|>\delta_{1}} \int_{\left|v_{2}-\theta_{1}^{2} h_{2}\right|>\delta_{2}} \mid v_{1} v_{2}\left(h_{1} h_{2}\right)^{-1} \mathbb{K}\left(\frac{v_{1}}{h_{1}}, \frac{v_{2}}{h_{2}}\right)\left(\left(h_{1} h_{2}\right)^{-1}\right. \\
& \left.\times \mathbb{K}\left(\frac{v_{1}+x_{1}-\theta_{1}^{1}-y_{1}+\theta_{2}^{1} h_{1}}{h_{1}}, \frac{v_{2}+x_{2}-\theta_{1}^{2}-y_{2}+\theta_{2}^{2} h_{2}}{h_{2}}\right)\right) \\
& \times \frac{f\left(x_{1}+v_{1}-\theta_{1}^{1} h_{1}, x_{2}+v_{2}-\theta_{1}^{2} h_{2}\right)}{v_{1} v_{2}} \mid d v_{1} d v_{2} .
\end{aligned}
$$

We get

$$
\begin{aligned}
\int_{\left|v_{1}-\theta_{1}^{1} h_{1}\right|>\delta_{1}} \int_{\left|v_{2}-\theta_{1}^{2} h_{2}\right|>\delta_{2}} & \leq \frac{2}{\delta_{1}-\theta_{1}^{1} h_{1}} \times \frac{2}{\delta_{2}-\theta_{1}^{2} h_{2}} \sup _{\left|v_{1}-\theta_{1}^{1} h_{1}\right|>\delta_{1}} \sup _{\left|v_{2}-\theta_{1}^{2} h_{2}\right|>\delta_{2}}\left|\frac{v_{1} v_{2}}{h_{1} h_{2}} \mathbb{K}\left(\frac{v_{1}}{h_{1}}, \frac{v_{2}}{h_{2}}\right)\right| \\
& \times \int_{\left|v_{1}-\theta_{1}^{1} h_{1}\right|>\delta_{1}} \int_{\left|v_{1}-\theta_{1}^{1} h_{1}\right|>\delta_{2}}\left(\left(h_{1} h_{2}\right)^{-1}\right. \\
& \left.\times \mathbb{K}\left(\frac{v_{1}+x_{1}-\theta_{1}^{1}-y_{1}+\theta_{2}^{1} h_{1}}{h_{1}}, \frac{v_{2}+x_{2}-\theta_{1}^{2}-y_{2}+\theta_{2}^{2} h_{2}}{h_{2}}\right)\right) \\
& \times\left|f\left(x_{1}+v_{1}-\theta_{1}^{1} h_{1}, x_{2}+v_{2}-\theta_{1}^{2} h_{2}\right)\right| d v_{1} d v_{2} .
\end{aligned}
$$


Let the change of variables defined by

$$
v_{i}+x_{i}-\theta_{1}^{i} h_{i}-y_{i}+\theta_{2}^{i} h_{i}=u_{i}, \quad i=1,2 .
$$

Then

$$
\begin{aligned}
\int_{\left|v_{1}-\theta_{1}^{1} h_{1}\right|>\delta_{1}} \int_{\left|v_{2}-\theta_{1}^{2} h_{2}\right|>\delta_{2}} & \leq \frac{2}{\delta_{1}-\theta_{1}^{1} h_{1}} \times \frac{2}{\delta_{2}-\theta_{1}^{2} h_{2}} \sup _{\left|v_{1}-\theta_{1}^{1} h_{1}\right|>\delta_{1}} \sup _{\left|v_{2}-\theta_{1}^{2} h_{2}\right|>\delta_{2}}\left|\frac{v_{1} v_{2}}{h_{1} h_{2}} \mathbb{K}\left(\frac{v_{1}}{h_{1}}, \frac{v_{2}}{h_{2}}\right)\right| \\
& \times \iint_{\mathbb{R}^{2}}\left|\left(h_{1} h_{2}\right)^{-1} \mathbb{K}\left(\frac{u_{1}}{h_{1}}, \frac{u_{2}}{h_{2}}\right)\right| f\left(u_{1}+y_{1}-\theta_{2}^{1} h_{1}, u_{2}+y_{2}-\theta_{2}^{2} h_{2}\right) d u_{1} d u_{2}
\end{aligned}
$$

For Lemma 4 (replacing $\mathbb{K}$ by $|\mathbb{K}|$ ) and $\left(\mathbf{H}_{\mathbf{3}}\right)$ we have

$$
\left|\int_{\left|v_{1}-\theta_{1}^{1} h_{1}\right|>\delta_{1}} \int_{\left|v_{1}-\theta_{1}^{1} h_{1}\right|>\delta_{2}}\right| \rightarrow 0 \text { as } n \rightarrow+\infty,
$$

and the convergence is uniform in this case.

Consider the case $\int_{\left|v_{1}-\theta_{1}^{1} h_{1}\right| \leq \delta_{1}} \int_{\left|v_{1}-\theta_{1}^{1} h_{1}\right|>\delta_{2}}(\cdot)(\cdot)$, by Fubini's theorem we have

$$
\int_{\left|v_{1}-\theta_{1}^{1} h_{1}\right| \leq \delta_{1}} \int_{\left|v_{1}-\theta_{1}^{1} h_{1}\right|>\delta_{2}}(\cdot)(\cdot)=\int_{\left|v_{1}-\theta_{1}^{1} h_{1}\right| \leq \delta_{1}}(\cdot) \int_{\left|v_{1}-\theta_{1}^{1} h_{1}\right|>\delta_{2}}(\cdot) .
$$

Using the two cases treated in one dimension (Lemma 2.3 Dia (2008) and Lemma 2.9 Ciss et al. (2014)), we get

$$
\int_{\left|v_{1}-\theta_{1}^{1} h_{1}\right| \leq \delta_{1}} \rightarrow 0 \text { and } \int_{\left|v_{1}-\theta_{1}^{1} h_{1}\right|>\delta_{2}} \rightarrow 0 \quad \text { as } n \rightarrow+\infty
$$

therefore

$$
\int_{\left|v_{1}-\theta_{1}^{1} h_{1}\right| \leq \delta_{1}} \int_{\left|v_{2}-\theta_{1}^{2} h_{2}\right|>\delta_{2}} \rightarrow 0 \quad \text { as } \quad n \rightarrow+\infty .
$$
have

Consider the case $\int_{\left|v_{1}-\theta_{1}^{1} h_{1}\right|>\delta_{1}} \int_{\left|v_{2}-\theta_{1}^{2} h_{2}\right| \leq \delta_{2}}(\cdot)(\cdot)$, similarly by Fubini's theorem we

$$
\int_{\left|v_{1}-\theta_{1}^{1} h_{1}\right|>\delta_{1}} \int_{\left|v_{2}-\theta_{1}^{2} h_{2}\right| \leq \delta_{2}}(\cdot)(\cdot)=\int_{\left|v_{1}-\theta_{1}^{1} h_{1}\right|>\delta_{1}}(\cdot) \int_{\left|v_{2}-\theta_{1}^{2} h_{2}\right| \leq \delta_{2}}(\cdot),
$$

therefore

$$
\int_{\left|v_{1}-\theta_{1}^{1} h_{1}\right|>\delta_{1}} \int_{\left|v_{2}-\theta_{1}^{2} h_{2}\right| \leq \delta_{2}} \rightarrow 0 \quad \text { as } \quad n \rightarrow+\infty .
$$

This complete the proof of Lemma 3. 
Remark 3. According to the case considered, if $C_{2}$ is verified then for $\int_{\left|v_{1}-\theta_{1}^{1} h_{1}\right|>\delta_{1}} \int_{\left|v_{1}-\theta_{1}^{1} h_{1}\right|>\delta_{2}}$, the integral of the right hand-side of 12 becomes

$$
\begin{aligned}
& \iint_{\mathbb{R}^{2}}\left|\left(h_{1} h_{2}\right)^{-1} \mathbb{K}\left(\frac{u_{1}}{h_{1}}, \frac{u_{2}}{h_{2}}\right)\right| \mid f\left(u_{1}+y_{1}-\theta_{2}^{1} h_{1}, u_{2}+y_{2}-\theta_{2}^{2} h_{2}\right) \\
& -f\left(\frac{u_{1}}{h_{1}}, \frac{u_{2}}{h_{2}}\right)+f\left(\frac{u_{1}}{h_{1}}, \frac{u_{2}}{h_{2}}\right) \mid d u_{1} d u_{2} \\
& \leq \iint_{\mathbb{R}^{2}}\left|\left(h_{1} h_{2}\right)^{-1} \mathbb{K}\left(\frac{u_{1}}{h_{1}}, \frac{u_{2}}{h_{2}}\right)\right| \int_{\frac{u_{1}}{h_{1}}}^{u_{1}+y_{1}-\theta_{2}^{1} h_{1}} \int_{\frac{u_{2}}{h_{2}}}^{u_{2}+y_{2}-\theta_{2}^{2} h_{2}}\left|f^{\prime}\left(t_{1}, t_{2}\right)\right| d t_{1} d t_{2} d u_{1} d u_{2} \\
& +\iint_{\mathbb{R}^{2}} f\left(\frac{u_{1}}{h_{1}}, \frac{u_{2}}{h_{2}}\right)\left|\left(h_{1} h_{2}\right)^{-1} \mathbb{K}\left(\frac{u_{1}}{h_{1}}, \frac{u_{2}}{h_{2}}\right)\right| d u_{1} d u_{2} \\
& \leq \iint_{\mathbb{R}^{2}}\left|\left(h_{1} h_{2}\right)^{-1} \mathbb{K}\left(\frac{u_{1}}{h_{1}}, \frac{u_{2}}{h_{2}}\right)\right| d u_{1} d u_{2} \iint_{\mathbb{R}^{2}}\left|f^{\prime}\left(t_{1}, t_{2}\right)\right| d t_{1} d t_{2} \\
& +\iint_{\mathbb{R}^{2}}\left|\mathbb{K}\left(u_{1}, u_{2}\right)\right| f\left(u_{1}, u_{2}\right) d u_{1} d u_{2} .
\end{aligned}
$$

The integrals of the right hand-side of this last inequality are bounded. Hence the theorem is valid under the hypothesis $C_{2}$.

For the cases $\int_{\left|v_{1}-\theta_{1}^{1} h_{1}\right| \leq \delta_{1}} \int_{\left|v_{1}-\theta_{1}^{1} h_{1}\right|>\delta_{2}}$ and $\int_{\left|v_{1}-\theta_{1}^{1} h_{1}\right|>\delta_{1}} \int_{\left|v_{1}-\theta_{1}^{1} h_{1}\right| \leq \delta_{2}}$ applying Fubini's theorem and the results of Lemma 2.3 Dia (2008) in the one-dimensional case we give us the result.

\subsection{Proof of Theorem 5}

We suppose condition $\quad \mathbf{C}_{\mathbf{1}}$ verified. Let $\Delta_{1}=\left[0, z_{1}\right] \times\left[0, z_{1}\right] ; \Delta_{2}=\left[0, z_{2}\right] \times\left[0, z_{2}\right]$. We can write

$$
\begin{aligned}
& \sum_{0 \leq i_{1} \neq j_{1} \leq\left[\frac{z_{1}}{h_{1}}\right]}\left(1-\frac{i_{1} h_{1}}{z_{1}}\right)^{\alpha_{1}}\left(1-\frac{j_{1} h_{1}}{z_{1}}\right)^{\alpha_{1}} \sum_{0 \leq i_{2} \neq j_{2} \leq\left[\frac{z_{2}}{h_{2}}\right]}\left(1-\frac{i_{2} h_{2}}{z_{2}}\right)^{\alpha_{2}}\left(1-\frac{j_{2} h_{2}}{z_{2}}\right)^{\alpha_{2}} \\
&\left.\times \iint_{\mathbb{R}^{2}}\left|\mathbb{K}\left(\frac{u_{1}-i_{1} h_{1}}{h_{1}}, \frac{u_{1}-j_{1} h_{1}}{h_{1}}\right) \mathbb{K}\left(\frac{u_{2}-i_{2} h_{2}}{h_{2}}, \frac{u_{2}-j_{2} h_{2}}{h_{2}}\right)\right| f\left(u_{1}, u_{2}\right) d u_{1} d u_{2}\right) \\
&=\iint_{\left\{\left(x_{1}, y_{1}\right) \in \Delta_{1}:\left|x_{1}-y_{1}\right|>0\right\}} \Phi_{n}\left(x_{1}, y_{1}\right) d x_{1} d y_{1} \iint_{\left\{\left(x_{2}, y_{2}\right) \in \Delta_{2}:\left|x_{2}-y_{2}\right|>0\right\}} \Phi_{n}\left(x_{2}, y_{2}\right) d x_{2} d y_{2},
\end{aligned}
$$

where

$$
\begin{aligned}
\Phi_{n}\left(x_{1}, y_{1}\right)=\frac{1}{h_{1}^{2}} \sum_{0 \leq i_{1} \neq j_{1} \leq\left[\frac{z_{1}}{h_{1}}\right]} \chi_{\Delta_{h_{1}, i_{1}} \times \Delta_{h_{1}, j_{1}}}\left(x_{1}, y_{1}\right)\left(1-\frac{i_{1} h_{1}}{z_{1}}\right)^{\alpha_{1}}\left(1-\frac{j_{1} h_{1}}{z_{1}}\right)^{\alpha_{1}} \\
\times \int_{\mathbb{R}}\left|\mathbb{K}\left(\frac{u_{1}-i_{1} h_{1}}{h_{1}}, \frac{u_{1}-j_{1} h_{1}}{h_{1}}\right)\right| f_{1}\left(u_{1}\right) d u_{1},
\end{aligned}
$$

and $\Phi_{n}\left(x_{2}, y_{2}\right)$ is obtained by replacing $x_{1}$ by $x_{2}, y_{1}$ by $y_{2}, u_{1}$ by $u_{2}$ and $f_{1}$ by $f_{2}$. 
Note $\Phi_{n_{12}}\left(x_{1}, y_{1}, x_{2}, y_{2}\right):=\Phi_{n}\left(x_{1}, y_{1}\right) \Phi_{n}\left(x_{2}, y_{2}\right)$. Therefore

$$
\begin{aligned}
\Phi_{n_{12}}\left(x_{1}, y_{1}, x_{2}, y_{2}\right)=\frac{1}{h_{1}^{2} h_{2}^{2}} \sum_{0 \leq i_{1} \neq j_{1} \leq\left[\frac{z_{1}}{h_{1}}\right]} \chi_{\Delta_{h_{1}, i_{1}} \times \Delta_{h_{1}, j_{1}}}\left(x_{1}, y_{1}\right)\left(1-\frac{i_{1} h_{1}}{z_{1}}\right)^{\alpha_{1}}\left(1-\frac{j_{1} h_{1}}{z_{1}}\right)^{\alpha_{1}} \\
\quad \times \sum_{0 \leq i_{2} \neq j_{2} \leq\left[\frac{z_{2}}{h_{2}}\right]} \chi_{\Delta_{h_{2}, i_{2}} \times \Delta_{h_{2}, j_{2}}}\left(x_{2}, y_{2}\right)\left(1-\frac{i_{2} h_{2}}{z_{2}}\right)^{\alpha_{2}}\left(1-\frac{j_{2} h_{2}}{z_{2}}\right)^{\alpha_{2}} \\
\times \iint_{\mathbb{R}^{2}}\left|\mathbb{K}\left(\frac{u_{1}-i_{1} h_{1}}{h_{1}}, \frac{u_{1}-j_{1} h_{1}}{h_{1}}\right) \mathbb{K}\left(\frac{u_{2}-i_{1} h_{2}}{h_{2}}, \frac{u_{2}-j_{2} h_{2}}{h_{2}}\right)\right| f\left(u_{1}, u_{2}\right) d u_{1} d u_{2} .
\end{aligned}
$$

If $\left(x_{1}, y_{1}\right) \in \Delta_{h_{1}, i_{1}} \times \Delta_{h_{1}, j_{1}} \quad i_{1} \neq j_{1} ; \quad\left(x_{2}, y_{2}\right) \in \Delta_{h_{2}, i_{2}} \times \Delta_{h_{2}, j_{2}} \quad i_{2} \neq j_{2} \quad$ with the representations

$$
x_{1}=h_{1} i_{1}+\theta_{1}^{1} h_{1}, \quad y_{1}=h_{1} j_{1}+\theta_{2}^{1} h_{1} \quad 0 \leq \theta_{i}^{1}<1, \quad i=1,2,
$$

and

We have

$$
x_{2}=h_{2} i_{2}+\theta_{1}^{2} h_{2}, \quad y_{2}=h_{2} j_{2}+\theta_{2}^{2} h_{2} \quad 0 \leq \theta_{i}^{2}<1, \quad i=1,2 .
$$

$$
\begin{aligned}
& \frac{1}{h_{1}^{2} h_{2}^{2}}\left(1-\frac{i_{1} h_{1}}{z_{1}}\right)^{\alpha_{1}}\left(1-\frac{j_{1} h_{1}}{z_{1}}\right)^{\alpha_{1}}\left(1-\frac{i_{2} h_{2}}{z_{2}}\right)^{\alpha_{2}}\left(1-\frac{j_{2} h_{2}}{z_{2}}\right)^{\alpha_{2}} \\
& \times \iint_{\mathbb{R}^{2}}\left|\mathbb{K}\left(\frac{u_{1}-x_{1}+\theta_{1}^{1} h_{1}}{h_{1}}, \frac{u_{1}-y_{1}+\theta_{2}^{1} h_{1}}{h_{1}}\right) \mathbb{K}\left(\frac{u_{2}-x_{2}+\theta_{1}^{2} h_{2}}{h_{2}}, \frac{u_{2}-y_{2}+\theta_{2}^{2} h_{2}}{h_{2}}\right)\right| \\
& \times f\left(u_{1}, u_{2}\right) d u_{1} d u_{2} \\
& \leq \frac{1}{h_{1}^{2} h_{2}^{2}} \iint_{\mathbb{R}^{2}}\left|\mathbb{K}\left(\frac{u_{1}-x_{1}+\theta_{1}^{1} h_{1}}{h_{1}}, \frac{u_{1}-y_{1}+\theta_{2}^{1} h_{1}}{h_{1}}\right) \mathbb{K}\left(\frac{u_{2}-x_{2}+\theta_{1}^{2} h_{2}}{h_{2}}, \frac{u_{2}-y_{2}+\theta_{2}^{2} h_{2}}{h_{2}}\right)\right| \\
& \times f\left(u_{1}, u_{2}\right) d u_{1} d u_{2} .
\end{aligned}
$$

The right hand-side tends to zero as $n \rightarrow+\infty$ according to the Lemma 3 .

Let $\delta_{i}=\frac{z_{i}}{2}, \quad i=1,2$. Write

$$
\begin{aligned}
& \frac{1}{h_{1}^{2} h_{2}^{2}} \iint_{\mathbb{R}^{2}}\left|\mathbb{K}\left(\frac{u_{1}-x_{1}+\theta_{1}^{1} h_{1}}{h_{1}}, \frac{u_{1}-y_{1}+\theta_{2}^{1} h_{1}}{h_{1}}\right) \mathbb{K}\left(\frac{u_{2}-x_{2}+\theta_{1}^{2} h_{2}}{h_{2}}, \frac{u_{2}-y_{2}+\theta_{2}^{2} h_{2}}{h_{2}}\right)\right| \\
& \times f\left(u_{1}, u_{2}\right) d u_{1} d u_{2} \\
& =\int_{\left|v_{1}\right| \leq \delta_{1}} \int_{\left|v_{2}\right| \leq \delta_{2}}+\int_{\left|v_{1}\right|>\delta_{1}} \int_{\left|v_{2}\right|>\delta_{2}}+\int_{\left|v_{1}\right| \leq \delta_{1}} \int_{\left|v_{2}\right|>\delta_{2}}+\int_{\left|v_{1}\right|>\delta_{1}} \int_{\left|v_{2}\right| \leq \delta_{2}} .
\end{aligned}
$$

Then, we have

$$
\begin{aligned}
& \iint_{\left\{\left(x_{1}, y_{1}\right) \in \Delta_{1}:\left|x_{1}-y_{1}\right|>0\right\}} \Phi_{n}\left(x_{1}, y_{1}\right) d x_{1} d y_{1} \iint_{\left\{\left(x_{2}, y_{2}\right) \in \Delta_{2}:\left|x_{2}-y_{2}\right|>0\right\}} \Phi_{n}\left(x_{2}, y_{2}\right) d x_{2} d y_{2} \\
& \leq \iint_{\left\{\left(x_{1}, y_{1}\right) \in \Delta_{1}:\left|x_{1}-y_{1}\right|>0\right\}} \sum_{0 \leq i_{1} \neq j_{1} \leq\left[\frac{z_{1}}{h_{1}}\right]} \chi_{\Delta_{h_{1}, i_{1}} \times \Delta_{h_{1}, j_{1}}}\left(x_{1}, y_{1}\right) \\
& \times \iint_{\left\{\left(x_{2}, y_{2}\right) \in \Delta_{2}:\left|x_{x} 2-y_{2}\right|>0\right\}} \sum_{0 \leq i_{2} \neq j_{2} \leq\left[\frac{z_{2}}{h_{2}}\right]} \chi_{\Delta_{h_{2}, i_{2}} \times \Delta_{h_{2}, j_{2}}}\left(x_{2}, y_{2}\right) \\
& \left(\int_{\left|v_{1}\right| \leq \delta_{1}} \int_{\left|v_{2}\right| \leq \delta_{2}}+\int_{\left|v_{1}\right|>\delta_{1}} \int_{\left|v_{2}\right|>\delta_{2}}+\int_{\left|v_{1}\right| \leq \delta_{1}} \int_{\left|v_{2}\right|>\delta_{2}}+\int_{\left|v_{1}\right|>\delta_{1}} \int_{\left|v_{2}\right| \leq \delta_{2}}\right) .
\end{aligned}
$$


The proof of the remainder is conducted as follow:

First consider

$$
\iint_{\left\{\left(x_{1}, y_{1}\right) \in \Delta_{1}:\left|x_{1}-y_{1}\right|>0\right\}} \iint_{\left\{\left(x_{2}, y_{2}\right) \in \Delta_{2}:\left|x_{2}-y_{2}\right|>0\right\}} \int_{\left|v_{1}\right| \leq \delta_{1}} \int_{\left|v_{2}\right| \leq \delta_{2}} .
$$

Define $A=\sup _{(x, y) \in\left[0, z_{1}\right] \times\left[0, z_{2}\right]} f(x, y)$. The notations being as in the proof Lemma 3 with $\delta_{i}=\frac{z_{i}}{2}, \quad i=1,2$, we have following inequality 10

$$
\begin{aligned}
& \int_{\left|v_{1}\right| \leq \delta_{1}} \int_{\left|v_{2}\right| \leq \delta_{2}} \leq A \int_{-\infty}^{+\infty} \chi_{-\frac{\delta_{1}}{h_{1}} \leq u_{1} \leq \frac{\delta_{1}}{h_{1}}} \int_{-\infty}^{+\infty} \chi_{-\frac{\delta_{2}}{h_{2}} \leq u_{2} \leq \frac{\delta_{2}}{h_{2}}}\left|\mathbb{K}\left(u_{1}, u_{2}\right)\right| \\
& \times\left|\mathbb{K}\left(\frac{x_{1}-\theta_{1}^{1} h_{1}-y_{1}+\theta_{2}^{1} h_{1}}{h_{1}}+u_{1}, \frac{x_{2}-\theta_{1}^{2} h_{2}-y_{2}+\theta_{2}^{2} h_{2}}{h_{2}}+u_{2}\right)\left(h_{1} h_{2}\right)^{-1}\right| d u_{1} d u_{2} .
\end{aligned}
$$

For all $\left(u_{1}, u_{2}\right)$,

$$
\lim _{n \rightarrow+\infty}\left|\mathbb{K}\left(\frac{x_{1}-\theta_{1}^{1} h_{1}-y_{1}+\theta_{2}^{1} h_{1}}{h_{1}}+u_{1}, \frac{x_{2}-\theta_{1}^{2} h_{2}-y_{2}+\theta_{2}^{2} h_{2}}{h_{2}}+u_{2}\right)\left(h_{1} h_{2}\right)^{-1}\right|=0 .
$$

Write

$$
\begin{aligned}
& \left|\mathbb{K}\left(\frac{x_{1}-\theta_{1}^{1} h_{1}-y_{1}+\theta_{2}^{1} h_{1}}{h_{1}}+u_{1}, \frac{x_{2}-\theta_{1}^{2} h_{2}-y_{2}+\theta_{2}^{2} h_{2}}{h_{2}}+u_{2}\right)\left(h_{1} h_{2}\right)^{-1}\right| \\
& =\mid \mathbb{K}\left(\frac{x_{1}-\theta_{1}^{1} h_{1}-y_{1}+\theta_{2}^{1} h_{1}}{h_{1}}+u_{1}, \frac{x_{2}-\theta_{1}^{2} h_{2}-y_{2}+\theta_{2}^{2} h_{2}}{h_{2}}+u_{2}\right) \\
& -\mathbb{K}\left(\frac{2 z_{1}+x_{1}-\theta_{1}^{1}-y_{1}+\theta_{2}^{1} h_{1}}{h_{1}}+u_{1}, \frac{z_{2}+x_{2}-\theta_{1}^{2}-y_{2}+\theta_{2}^{2} h_{2}}{h_{2}}+u_{2}\right) \\
& +\mathbb{K}\left(\frac{2 z_{1}+x_{1}-\theta_{1}^{1}-y_{1}+\theta_{2}^{1} h_{1}}{h_{1}}+u_{1}, \frac{z_{2}+x_{2}-\theta_{1}^{2}-y_{2}+\theta_{2}^{2} h_{2}}{h_{2}}+u_{2}\right) \mid\left(h_{1} h_{2}\right)^{-1} \\
& \leq\left(\lambda\left(\frac{2 z_{1}}{h_{1}}, \frac{2 z_{2}}{h_{2}}\right)+\left|\mathbb{K}\left(\frac{2 z_{1}+x_{1}-\theta_{1}^{1} h_{1}-y_{1}+\theta_{2}^{1} h_{1}}{h_{1}}+u_{1}, \frac{z_{2}+x_{2}-\theta_{1}^{2}-y_{2}+\theta_{2}^{2} h_{2}}{h_{2}}+u_{2}\right)\right|\right) \\
& \times\left(h_{1} h_{2}\right)^{-1} .
\end{aligned}
$$

Moreover,

$$
\begin{aligned}
& \left|\mathbb{K}\left(\frac{2 z_{1}+x_{1}-\theta_{1}^{1}-y_{1}+\theta_{2}^{1} h_{1}}{h_{1}}+u_{1}, \frac{z_{2}+x_{2}-\theta_{1}^{2}-y_{2}+\theta_{2}^{2} h_{2}}{h_{2}}+u_{2}\right)\right|\left(h_{1} h_{2}\right)^{-1} \\
& =\left|\frac{2 z_{1}+x_{1}-y_{1}+h_{1} u_{1}}{h_{1}}\right|\left|\frac{2 z_{2}+x_{2}-y_{2}+h_{2} u_{2}}{h_{2}}\right| \\
& \times\left|\mathbb{K}\left(\frac{2 z_{1}+x_{1}-\theta_{1}^{1}-y_{1}+\theta_{2}^{1} h_{1}}{h_{1}}+u_{1}, \frac{z_{2}+x_{2}-\theta_{1}^{2}-y_{2}+\theta_{2}^{2} h_{2}}{h_{2}}+u_{2}\right)\right| \\
& \times \frac{1}{\left|2 z_{1}+x_{1}-y_{1}+h_{1} u_{1}\right|\left|2 z_{2}+x_{2}-y_{2}+h_{2} u_{2}\right|} .
\end{aligned}
$$


We have

$$
\begin{aligned}
T T & =\left|\frac{2 z_{1}+x_{1}-y_{1}+h_{1} u_{1}}{h_{1}}\right|\left|\frac{2 z_{2}+x_{2}-y_{2}+h_{2} u_{2}}{h_{2}}\right| \\
& =\left|\frac{2 z_{1}+x_{1}-y_{1}+h_{1} u_{1}-\theta_{1}^{1} h_{1}+\theta_{2}^{1} h_{1}}{h_{1}}+\frac{\theta_{1}^{1} h_{1}-\theta_{2}^{1} h_{1}}{h_{1}}\right| \\
& \times\left|\frac{2 z_{2}+x_{2}-y_{2}+h_{2} u_{2}-\theta_{1}^{2} h_{2}+\theta_{2}^{2} h_{2}}{h_{2}}+\frac{\theta_{2}^{2} h_{2}-\theta_{2}^{2} h_{2}}{h_{2}}\right| \\
& \leq\left|\frac{2 z_{1}+x_{1}-y_{1}+h_{1} u_{1}-\theta_{1}^{1} h_{1}+\theta_{2}^{1} h_{1}}{h_{1}}\right|+\left|\frac{\theta_{1}^{1} h_{1}-\theta_{2}^{1} h_{1}}{h_{1}}\right| \\
& \left.\times\left|\frac{2 z_{2}+x_{2}-y_{2}+h_{2} u_{2}-\theta_{1}^{2} h_{2}+\theta_{2}^{2} h_{2}}{h_{2}}\right|+\mid \frac{\theta_{1}^{2} h_{2}-\theta_{2}^{2} h_{2}}{h_{2}}\right) \mid \\
& \leq\left|\frac{2 z_{1}+x_{1}-y_{1}+h_{1} u_{1}-\theta_{1}^{1} h_{1}+\theta_{2}^{1} h_{1}}{h_{1}}\right|\left|\frac{2 z_{2}+x_{2}-y_{2}+h_{2} u_{2}-\theta_{1}^{2} h_{2}+\theta_{2}^{2} h_{2}}{h_{2}}\right| \\
& +\left|\frac{2 z_{1}+x_{1}-y_{1}+h_{1} u_{1}-\theta_{1}^{1} h_{1}+\theta_{2}^{1} h_{1}}{h_{1}}\right|+\left|\frac{2 z_{2}+x_{2}-y_{2}+h_{2} u_{2}-\theta_{1}^{2} h_{2}+\theta_{2}^{2} h_{2}}{h_{2}}\right|+1 .
\end{aligned}
$$

Let $B=\sup _{y \in \mathbb{R}}|y||K(y)|$ and $C=\sup _{y \in \mathbb{R}}|K(y)|$, then we have

$$
\begin{gathered}
\left|\frac{2 z_{1}+x_{1}-y_{1}+h_{1} u_{1}}{h_{1}} \| \frac{2 z_{2}+x_{2}-y_{2}+h_{2} u_{2}}{h_{2}}\right| \mid K\left(\frac{2 z_{1}+x_{1}-\theta_{1}^{1}-y_{1}+\theta_{2}^{1} h_{1}}{h_{1}}+u_{1}\right) \\
\times K\left(\frac{z_{2}+x_{2}-\theta_{1}^{2}-y_{2}+\theta_{2}^{2} h_{2}}{h_{2}}+u_{2}\right) \mid \\
\leq B^{2}+2 B C+C
\end{gathered}
$$

Therefore we have

$$
\begin{aligned}
& \mid K\left(\frac{2 z_{1}+x_{1}-\theta_{1}^{1}-y_{1}+\theta_{2}^{1} h_{1}}{h_{1}}+u_{1}\right) K(\left.\frac{z_{2}+x_{2}-\theta_{1}^{2}-y_{2}+\theta_{2}^{2} h_{2}}{h_{2}}+u_{2}\right) \mid\left(h-1 h_{2}\right)^{-1} \\
& \leq \frac{B^{2}+2 B C+C}{\left|2 z_{1}+x_{1}-y_{1}+h_{1} u_{1}\right|\left|2 z_{2}+x_{2}-y_{2}+h_{2} u_{2}\right|}
\end{aligned}
$$

hence

$$
\begin{aligned}
\left|\mathbb{K}\left(\frac{x_{1}-\theta_{1}^{1} h_{1}-y_{1}+\theta_{2}^{1} h_{1}}{h_{1}}+u_{1}, \frac{x_{2}-\theta_{1}^{2} h_{2}-y_{2}+\theta_{2}^{2} h_{2}}{h_{2}}+u_{2}\right)\left(h_{1} h_{2}\right)^{-1}\right| \\
\leq\left(\lambda\left(\frac{2 z_{1}}{h_{1}}, \frac{2 z_{2}}{h_{2}}\right)+\frac{B^{2}+2 B C+C}{\left|2 z_{1}+x_{1}-y_{1}+h_{1} u_{1}\right|\left|2 z_{2}+x_{2}-y_{2}+h_{2} u_{2}\right|} .\right.
\end{aligned}
$$

Journal home page: www.jafristat.net 
We conclude that for $h_{i}, \quad i=1,2$, small enough

$$
\begin{aligned}
& \int_{\left|v_{1}\right| \leq \delta_{1}} \int_{\left|v_{2}\right| \leq \delta_{2}} \leq \frac{A}{\left|2 z_{1}+x_{1}-y_{1}+h_{1} u_{1}\right|\left|2 z_{2}+x_{2}-y_{2}+h_{2} u_{2}\right|} \\
& \times \iint_{\mathbb{R}^{2}}\left|\mathbb{K}\left(u_{1}, u_{2}\right)\right|\left(B^{2}+2 B C+C\right) d u_{1} d u_{2} \\
&<\frac{A D}{\left|2 z_{1}+x_{1}-y_{1}+h_{1} u_{1}\right|\left|2 z_{2}+x_{2}-y_{2}+h_{2} u_{2}\right|} \\
& \leq \frac{A D}{\left|2 z_{1}+x_{1}-y_{1}+h_{1} u_{1}\right|\left|2 z_{2}+x_{2}-y_{2}+h_{2} u_{2}\right|} \\
& \leq \frac{A D}{\left(2 z_{1}+x_{1}-y_{1}+h_{1} u_{1}\right)\left(2 z_{2}+x_{2}-y_{2}+h_{2} u_{2}\right)}
\end{aligned}
$$

where $D$ being the finite bound of $\iint_{\mathbb{R}^{2}}\left|\mathbb{K}\left(u_{1}, u_{2}\right)\right|\left(B^{2}+2 B C+4 C\right) d u_{1} d u_{2}$.

Finally, we have

$$
\int_{\left|v_{1}\right| \leq \delta_{1}} \int_{\left|v_{2}\right| \leq \delta_{2}} \leq \frac{A D}{\left(2 z_{1}+x_{1}-y_{1}+h_{1} u_{1}\right)\left(2 z_{2}+x_{2}-y_{2}+h_{2} u_{2}\right)}+O\left(h_{1} h_{2}\right) .
$$

Since $-\delta_{i} \leq h_{1} u_{1} \leq \delta_{i}$, we have $\frac{z_{i}}{2} \leq 2 z_{i}+x_{i}-y_{i}+h_{i} u_{i} \leq \frac{7 z_{i}}{2}, \quad i=1,2$, we get

$$
\int_{\left|v_{1}\right| \leq \delta_{1}} \int_{\left|v_{2}\right| \leq \delta_{2}} \leq \frac{2 A D}{z_{1} z_{2}}+O\left(h_{1} h_{2}\right)
$$

Therefore by Lebesgue-dominated convergence theorem, we have

$$
\begin{aligned}
\lim _{n \rightarrow+\infty} \iint_{\Delta_{1}} \iint_{\Delta_{2}}\left(\int_{\left|v_{1}\right| \leq \delta_{1}}\right. & \left.\int_{\left|v_{2}\right| \leq \delta_{2}}\right) d x_{1} d x_{2} d y_{1} d y_{2} \\
& =\iint_{\Delta_{1}} \iint_{\Delta_{2}} \lim _{n \rightarrow+\infty}\left(\int_{\left|v_{1}\right| \leq \delta_{1}} \int_{\left|v_{2}\right| \leq \delta_{2}}\right) d x_{1} d x_{2} d y_{1} d y_{2}=0 .
\end{aligned}
$$

Consider then $\int_{\left|v_{1}\right|>\delta_{1}} \int_{\left|v_{2}\right|>\delta_{2}}$.

We use the second part, by analogous reasoning, of the proof of Lemma 3

$$
\begin{aligned}
\int_{\left|v_{1}\right|>\delta_{1}} \int_{\left|v_{2}\right|>\delta_{2}} & \leq \frac{2}{\delta_{1}} \times \frac{2}{\delta_{2}} \sup _{\left|v_{1}\right|>\delta_{1}} \sup _{\left|v_{2}\right|>\delta_{2}}\left|\frac{v_{1}}{h_{1}} \frac{v_{2}}{h_{2}}\right| \mathbb{K}\left(\frac{v_{1}}{h_{1}}, \frac{v_{2}}{h_{2}}\right) \mid \\
& \times \iint_{\mathbb{R}^{2}}\left|\left(h_{1} h_{2}\right)^{-1} \mathbb{K}\left(\frac{u_{1}}{h_{1}}, \frac{u_{2}}{h_{2}}\right)\right| f\left(u_{1}+y_{1}-\theta_{2}^{1} h_{1}, u_{2}+y_{2}-\theta_{2}^{2} h_{2}\right) d u_{1} d u_{2}
\end{aligned}
$$

hence

$$
\int_{\left|v_{1}\right|>\delta_{1}} \int_{\left|v_{2}\right|>\delta_{2}} \rightarrow 0, \quad n \rightarrow+\infty
$$

uniformly. 
Consider $\int_{\left|v_{1}\right| \leq \delta_{1}} \int_{\left|v_{2}\right|>\delta_{2}}(\cdot)$ by Fubini's theorem we get

$$
\int_{\left|v_{1}\right| \leq \delta_{1}} \int_{\left|v_{2}\right|>\delta_{2}}(\cdot)=\int_{\left|v_{1}\right| \leq \delta_{1}}(\cdot) \int_{\left|v_{2}\right|>\delta_{2}}(\cdot) \rightarrow 0 * 0=0, \quad n \rightarrow+\infty
$$

according to the Lemma 2.3 for the one-dimensional case.

Similarly we have

$$
\int_{\left|v_{1}\right|>\delta_{1}} \int_{\left|v_{2}\right| \leq \delta_{2}}(\cdot)=\int_{\left|v_{1}\right|>\delta_{1}}(\cdot) \int_{\left|v_{2}\right| \leq \delta_{2}}(\cdot) \rightarrow 0 \times 0=0, \quad n \rightarrow+\infty .
$$

Consequently

$$
\lim _{n \rightarrow+\infty} \iint_{\Delta_{1}} \iint_{\Delta_{2}} \iint_{\mathbb{R}^{2}} \rightarrow 0, \quad n \rightarrow+\infty
$$

since $\Delta_{1}$ and $\Delta_{2}$ are bounded. The proof of the lemma is complete.

Remark 4. If $C_{2}$ is verified then the Theorem is again valid. Indeed it suffices to apply Remark 3 to inequality 13 in the case $\int_{\left|v_{1}\right|>\delta_{1}} \int_{\left|v_{2}\right|>\delta_{2}}$.

For the case $\int_{\left|v_{1}\right| \leq \delta_{1}} \int_{\left|v_{2}\right|>\delta_{2}}$ (resp. $\int_{\left|v_{1}\right|>\delta_{1}} \int_{\left|v_{2}\right| \leq \delta_{2}}$ ), apply Remark 3 to $\int_{\left|v_{2}\right|>\delta_{2}}$ ( resp. $\left.\int_{\left|v_{1}\right|>\delta_{1}}\right)$.

\subsection{Proof of Theorem 3}

We suppose condition $\left(\mathbf{C}_{\mathbf{1}}\right)$ satisfied, then

$$
\begin{aligned}
n \mathbb{V} a r\left(P_{n}\left(z_{1}, z_{2}, \alpha_{1}, \alpha_{2}\right)\right) & =\mathbb{E}\left(\sum_{i=0}^{\left[\frac{z_{1}}{h_{1}}\right]\left[\frac{z_{2}}{h_{2}}\right]} \sum_{j=0}^{i \frac{z_{1}}{h_{1}}\left[\frac{z_{2}}{h_{2}}\right]}\left(1-\frac{j h_{1}}{z_{1}}\right)^{\alpha_{1}}\left(1-\frac{\alpha_{2}}{z_{2}}\right)^{\alpha_{2}} K\left(\frac{X_{k}-i h_{1}}{h_{1}}\right) K\left(\frac{Y_{k}-j h_{2}}{h_{2}}\right)\right)^{2} \\
& -\mathbb{E}^{2}\left(\sum_{i=0}^{\alpha_{1}} \sum_{j=0}^{\alpha_{1}}\left(1-\frac{j h_{2}}{z_{1}}\right)^{\alpha_{2}} K\left(\frac{X_{k}-i h_{1}}{z_{1}}\right) K\left(\frac{Y_{k}-j h_{2}}{h_{2}}\right)\right) .
\end{aligned}
$$


Since

$$
\begin{aligned}
& \mathbb{E}\left(\sum_{i=0}^{\left[\frac{z_{1}}{h_{1}}\right]} \sum_{j=0}^{\left[\frac{z_{2}}{h_{2}}\right]}\left(1-\frac{i h_{1}}{z_{1}}\right)^{\alpha_{1}}\left(1-\frac{j h_{2}}{z_{2}}\right)^{\alpha_{2}} K\left(\frac{X_{k}-i h_{1}}{h_{1}}\right) K\left(\frac{Y_{k}-j h_{2}}{h_{2}}\right)\right)^{2} \\
= & \mathbb{E}\left[\left(\sum_{i=0}^{\left[\frac{z_{1}}{h_{1}}\right]}\left(1-\frac{i h_{1}}{z_{1}}\right)^{\alpha_{1}} K\left(\frac{X_{k}-i h_{1}}{h_{1}}\right)\right)^{2}\left(\sum_{j=0}^{\left[\frac{z_{2}}{h_{2}}\right]}\left(1-\frac{j h_{2}}{z_{2}}\right)^{\alpha_{2}} K\left(\frac{Y_{k}-j h_{2}}{h_{2}}\right)\right)^{2}\right] \\
= & \mathbb{E}\left[\left\{\sum_{i=0}^{\left[\frac{z_{1}}{h_{1}}\right]}\left(1-\frac{i h_{1}}{z_{1}}\right)^{2 \alpha_{1}} K^{2}\left(\frac{X_{k}-i h_{1}}{h_{1}}\right)\right.\right. \\
+ & \left.\sum_{i \neq l_{1}}^{\left[\frac{z_{1}}{h_{1}}\right]}\left(1-\frac{i h_{1}}{z_{1}}\right)^{\alpha_{1}}\left(1-\frac{l_{1} h_{1}}{z_{1}}\right)^{\alpha_{1}} K\left(\frac{X_{k}-i h_{1}}{h_{1}}\right) K\left(\frac{X_{k}-l_{1} h_{1}}{h_{1}}\right)\right\} \\
\times & \left\{\sum_{j=0}^{\left[\frac{z_{2}}{h_{2}}\right]}\left(1-\frac{j h_{2}}{z_{2}}\right)^{2 \alpha_{2}} K^{2}\left(\frac{Y_{k}-j h_{2}}{h_{2}}\right)\right. \\
& {\left[\frac{z_{2}}{h_{2}}\right] } \\
+ & \left.\left.\sum_{j \neq l_{2}}\left(1-\frac{j h_{2}}{z_{2}}\right)^{\alpha_{2}}\left(1-\frac{l_{2} h_{2}}{z_{2}}\right)^{\alpha_{2}} K\left(\frac{Y_{k}-j h_{2}}{h_{2}}\right) K\left(\frac{Y_{k}-l_{2} h_{2}}{h_{2}}\right)\right\}\right] \\
= & \mathbb{E}\left[m_{1} m_{3}+m_{1} m_{4}+m_{2} m_{3}+m_{2} m_{4}\right] .
\end{aligned}
$$

where

$$
\begin{aligned}
m_{1}:= & \sum_{i=0}^{\left[\frac{z_{1}}{h_{1}}\right]}\left(1-\frac{i h_{1}}{z_{1}}\right)^{2 \alpha_{1}} K^{2}\left(\frac{X_{k}-i h_{1}}{h_{1}}\right), \\
m_{2}:= & \sum_{i \neq l_{1}}^{\left[\frac{z_{1}}{h_{1}}\right]}\left(1-\frac{i h_{1}}{z_{1}}\right)^{\alpha_{1}}\left(1-\frac{l_{1} h_{1}}{z_{1}}\right)^{\alpha_{1}} K\left(\frac{X_{k}-i h_{1}}{h_{1}}\right) K\left(\frac{X_{k}-l_{1} h_{1}}{h_{1}}\right), \\
m_{3}:= & \sum_{j=0}^{\left[\frac{z_{2}}{h_{2}}\right]}\left(1-\frac{j h_{2}}{z_{2}}\right)^{2 \alpha_{2}} K^{2}\left(\frac{Y_{k}-j h_{2}}{h_{2}}\right), \\
m_{4}:= & \sum_{j \neq l_{2}}^{\left[\frac{z_{2}}{h_{2}}\right]}\left(1-\frac{j h_{2}}{z_{2}}\right)^{\alpha_{2}}\left(1-\frac{l_{2} h_{2}}{z_{2}}\right)^{\alpha_{2}} K\left(\frac{Y_{k}-j h_{2}}{h_{2}}\right) K\left(\frac{Y_{k}-l_{2} h_{2}}{h_{2}}\right) .
\end{aligned}
$$

We have, by the Theorem $5 \mathbb{E}\left(m_{2} m_{4}\right) \rightarrow 0, \quad$ as $n \rightarrow+\infty$.

According to Fubini, and the Theorem 2.5 Dia (2008) and Theorem 2.5 Ciss et al. (2014) in the unidimensional case, $\mathbb{E}\left(m_{1} m_{4}\right) \rightarrow 0$, and $\mathbb{E}\left(m_{2} m_{3}\right) \rightarrow 0, \quad$ as $n \rightarrow+\infty$.

On the other hand by Corollary 1 ,

$$
\mathbb{E}\left(m_{1} m_{3}\right) \rightarrow P\left(z_{1}, z_{2}, 2 \alpha_{1}, 2 \alpha_{2}\right) \int_{\mathbb{R}^{2}} \mathbb{K}^{2}\left(y_{1}, y_{2}\right)
$$


therefore

$$
n \mathbb{V} a r\left(P_{n}\left(z_{1}, z_{2}, \alpha_{1}, \alpha_{2}\right)\right) \rightarrow P\left(z_{1}, z_{2}, \alpha_{1}, \alpha_{2}\right) \int_{\mathbb{R}^{2}} \mathbb{K}^{2}\left(y_{1}, y_{2}\right)-P^{2}\left(z_{1}, z_{2}, \alpha_{1}, \alpha_{2}\right) .
$$

According to the Lemma 1 we have

$$
\mathbb{E}\left(P_{n}\left(z_{1}, z_{2}, \alpha_{1}, \alpha_{2}\right)\right) \rightarrow P\left(z_{1}, z_{2}, \alpha_{1}, \alpha_{2}\right) \text { as } n \rightarrow+\infty
$$

therefore

$$
\mathbb{E}^{2}\left(P_{n}\left(z_{1}, z_{2}, \alpha_{1}, \alpha_{2}\right)\right) \rightarrow P^{2}\left(z_{1}, z_{2}, \alpha_{1}, \alpha_{2}\right) \quad \text { as } n \rightarrow+\infty
$$

Define

$$
\operatorname{biais}\left(P_{n}\left(z_{1}, z_{2}, \alpha_{1}, \alpha_{2}\right)\right)=\mathbb{E}\left(P_{n}\left(z_{1}, z_{2}, \alpha_{1}, \alpha_{2}\right)\right)-P\left(z_{1}, z_{2}, \alpha_{1}, \alpha_{2}\right) \text {. }
$$

We have

$$
\begin{aligned}
& \left.\mathbb{E}\left(P_{n}\left(z_{1}, z_{2}, \alpha_{1}, \alpha_{2}\right)-P_{(} z_{1}, z_{2}, \alpha_{1}, \alpha_{2}\right)\right)^{2} \\
& =\operatorname{biais}^{2}\left(P_{n}\left(z_{1}, z_{2}, \alpha_{1}, \alpha_{2}\right)\right)+\operatorname{Var}\left(P_{n}\left(z_{1}, z_{2}, \alpha_{1}, \alpha_{2}\right)\right),
\end{aligned}
$$

and

$$
\left|\int_{\mathbb{R}^{2}} \mathbb{K}^{2}\left(y_{1}, y_{2}\right) P\left(z_{1}, z_{2}, \alpha_{1}, \alpha_{2}\right)-P^{2}\left(z_{1}, z_{2}, \alpha_{1}, \alpha_{2}\right)\right| \leq \int_{\mathbb{R}^{2}} \mathbb{K}^{2}\left(y_{1}, y_{2}\right)+1
$$

Hence

$$
\operatorname{Var}\left(P_{n}\left(z_{1}, z_{2}, \alpha_{1}, \alpha_{2}\right)\right)=O\left(\frac{1}{n}\right)
$$

By Lemma 1, we have

$$
\left|\mathbb{E}\left(P_{n}\left(z_{1}, z_{2}, \alpha_{1}, \alpha_{2}\right)\right)-P\left(z_{1}, z_{2}, \alpha_{1}, \alpha_{2}\right)\right| \rightarrow 0, \quad n \rightarrow+\infty,
$$

therefore

$$
\operatorname{biais}^{2}\left(P_{n}\left(z_{1}, z_{2}, \alpha_{1}, \alpha_{2}\right)\right) \rightarrow 0, \quad n \rightarrow+\infty
$$

hence

$$
\mathbb{E}\left(P_{n}\left(z_{1}, z_{2}, \alpha_{1}, \alpha_{2}\right)-P\left(z_{1}, z_{2}, \alpha_{1}, \alpha_{2}\right)\right)^{2} \rightarrow 0, \quad n \rightarrow+\infty .
$$

If condition $C_{2}$ is satisfied, the theorem is valid again, by Corollary 1, of Lemma 2 and using Remark 4 of Theorem 5 and by Theorem 2, we obtain the result of Theorem 4 .

\section{Appendix : Construction of the estimator based on the Riemann sums}

For $z_{1}>0, z_{2}>0$, and $h_{1}$ (resp. $\left.h_{2}\right)$ the length of the subdivisions for $\left[0, z_{1}\right]$ (resp. $\left.\left[0, z_{2}\right]\right)$, let $\Delta_{h_{1}, i}=\left[h_{1} i, h_{1}(i+1)\left[, i=0, \ldots,\left[\frac{z_{1}}{h_{1}}\right]-1,\left(\operatorname{resp} . \Delta_{h_{2}, j}=\left[h_{2} j, h_{2}(j+1)\left[, j=0, \ldots,\left[\frac{z_{2}}{h_{2}}\right]-1\right)\right.\right.\right.\right.$ be a partition of $\left[0, z_{1}\right]$, (resp. $\left.\left[0, z_{2}\right]\right)$. By the Riemann sum definition of the integral, we have that the limit of the following Riemann sum, say $\mathbf{I}_{n}$, over the two-dimensional rectangle $\left[0, z_{1}\right] \times\left[0, z_{2}\right]:$ 


$$
\begin{aligned}
& \mathbf{I}_{n}=\frac{1}{n} \sum_{k=1}^{n} \sum_{i=0}^{\left[\frac{z_{1}}{h_{1}}\right]-1} \sum_{j=0}^{\left[\frac{z_{2}}{h_{2}}\right]-1}\left(1-\frac{i h_{1}}{z_{1}}\right)^{\alpha_{1}}\left(1-\frac{j h_{1}}{z_{1}}\right)^{\alpha_{2}} \times \\
& K\left(\frac{X_{k}-i h_{1}}{h_{1}}\right) K\left(\frac{Y_{k}-i h_{2}}{h_{2}}\right)+\left(z_{1}-h_{1}\left[\frac{z_{1}}{h_{1}}\right]\right) \times \\
& \left(z_{2}-h_{2}\left[\frac{z_{2}}{h_{2}}\right]\right)\left(1-\frac{h_{1}\left[\frac{z_{1}}{h_{1}}\right]}{z_{1}}\right)^{\alpha_{1}}\left(1-\frac{h_{2}\left[\frac{z_{2}}{h_{2}}\right]}{z_{2}}\right)^{\alpha_{2}} \\
& \times \frac{1}{n} \sum_{k=1}^{n} \frac{1}{h_{1} h_{2}} K\left(\frac{X_{k}-\left[\frac{z_{1}}{h_{1}}\right] h_{1}}{h_{1}}\right) K\left(\frac{Y_{k}-\left[\frac{z_{1}}{h_{1}}\right] h_{2}}{h_{2}}\right) \\
& +\frac{1}{n h_{1}} \sum_{k=1}^{n} \sum_{j=0}^{\left[\frac{z_{2}}{h_{2}}\right]-1}\left(z_{1}-h_{1}\left[\frac{z_{1}}{h_{1}}\right]\right)\left(1-\frac{h_{1}\left[\frac{z_{1}}{h_{1}}\right]}{z_{1}}\right)^{\alpha_{1}} \times \\
& \left(1-\frac{j h_{2}}{z_{2}}\right)^{\alpha_{2}} K\left(\frac{X_{k}-\left[\frac{z_{1}}{h_{1}}\right] h_{1}}{h_{1}}\right) K\left(\frac{Y_{k}-j h_{2}}{h_{2}}\right) \\
& +\frac{1}{n h_{2}} \sum_{k=1}^{n} \sum_{i=0}^{\left[\frac{z_{1}}{h_{1}}\right]-1}\left(z_{2}-h_{2}\left[\frac{z_{2}}{h_{2}}\right]\right)\left(1-\frac{h_{2}\left[\frac{z_{2}}{h_{2}}\right]}{z_{2}}\right)^{\alpha_{2}} \times \\
& \left(1-\frac{i h_{1}}{z_{1}}\right)^{\alpha_{1}} K\left(\frac{X_{k}-i h_{1}}{h_{1}}\right) K\left(\frac{Y_{k}-\left[\frac{z_{2}}{h_{2}} h_{2}\right.}{h_{2}}\right)
\end{aligned}
$$

corresponding to the integral, say $\mathbf{I}$ :

$$
\mathbf{I}=\int_{0}^{z_{1}} \int_{0}^{z_{2}}\left(\frac{z_{1}-x}{z_{1}}\right)^{\alpha_{1}}\left(\frac{z_{2}-y}{z_{2}}\right)^{\alpha_{2}} \frac{1}{n} \sum_{i=1}^{n} \frac{1}{h_{1} h_{2}} K\left(\frac{x-X_{i}}{h_{1}}\right) K\left(\frac{x-Y_{i}}{h_{2}}\right) d x d y
$$

i.e., $\mathbf{I}=\lim _{n \rightarrow \infty} \mathbf{I}_{\mathbf{n}}$. This sum can be written as:

$$
\begin{array}{r}
\mathbf{I}_{n}=\frac{1}{n} \sum_{k=1}^{n} \sum_{i=0}^{\left[\frac{z_{1}}{h_{1}}\right]\left[\frac{z_{2}}{h_{2}}\right]} \sum_{j=0}\left(1-\frac{i h_{2}}{z_{2}}\right)^{\alpha_{1}}\left(1-\frac{j h_{2}}{z_{2}}\right)^{\alpha_{2}} \times \\
K\left(\frac{X_{k}-i h_{1}}{h_{1}}\right) K\left(\frac{Y_{k}-i h_{2}}{h_{2}}\right)+\mathcal{V}_{n}\left(z_{1}, z_{2}\right)+\mathcal{W}_{n}\left(z_{1}, z_{2}\right)+\mathcal{U}_{n}\left(z_{1}, z_{2}\right),
\end{array}
$$

where

$$
\begin{gathered}
\mathcal{V}_{n}\left(z_{1}, z_{2}\right)=\frac{1}{n} \frac{\left(z_{1}-h_{1}\left[\frac{z_{1}}{h_{1}}\right]\right)\left(z_{2}-h_{2}\left[\frac{z_{2}}{h_{2}}\right]\right)-h_{1} h_{2}}{h_{1} h_{2}}\left(1-\frac{h_{1}\left[\frac{z_{1}}{h_{1}}\right]}{z_{1}}\right)^{\alpha_{1}} \\
\times\left(1-\frac{h_{2}\left[\frac{z_{2}}{h_{2}}\right]}{z_{2}}\right)^{\alpha_{2}} \sum_{k=1}^{n} K\left(\frac{X_{k}-\left[\frac{z_{1}}{h_{1}}\right] h_{1}}{h_{1}}\right) K\left(\frac{Y_{k}-\left[\frac{z_{1}}{h_{1}}\right] h_{2}}{h_{2}}\right), \\
\mathcal{W}_{n}\left(z_{1}, z_{2}\right)=\frac{1}{n} \sum_{k=1}^{n} \sum_{j=0}^{\left[\frac{z_{2}}{h_{2}}\right]-1}\left(1-\frac{h_{1}\left[\frac{z_{1}}{h_{1}}\right]}{z_{1}}\right)^{\alpha_{1}}\left(1-\frac{j h_{2}}{z_{2}}\right)^{\alpha_{2}} \\
\times K\left(\frac{X_{k}-\left[\frac{z_{1}}{h_{1}}\right] h_{1}}{h_{1}}\right) K\left(\frac{Y_{k}-j h_{2}}{h_{2}}\right)\left(\frac{1}{h_{1}}\left(z_{1}-h_{1}\left[\frac{z_{1}}{h_{1}}\right]\right)-1\right),
\end{gathered}
$$




$$
\begin{aligned}
\mathcal{U}_{n}\left(z_{1}, z_{2}\right)=\frac{1}{n} \sum_{k=1}^{n} \sum_{0=1}^{\left[\frac{z_{1}}{h_{1}}\right]-1}\left(1-\frac{h_{2}\left[\frac{z_{2}}{h_{2}}\right]}{z_{2}}\right)^{\alpha_{2}}\left(1-\frac{i h_{1}}{z_{1}}\right)^{\alpha_{1}} & \\
& \times K\left(\frac{Y_{k}-\left[\frac{z_{2}}{h_{2}}\right] h_{2}}{h_{2}}\right) K\left(\frac{X_{k}-i h_{1}}{h_{1}}\right)\left(\frac{1}{h_{2}}\left(z_{2}-h_{2}\left[\frac{z_{2}}{h_{2}}\right]\right)-1\right) .
\end{aligned}
$$

Now it comes up that

$$
\mathcal{V}_{n} \longrightarrow 0, \quad \mathcal{W}_{n} \longrightarrow 0, \quad \mathcal{U}_{n} \longrightarrow 0, \quad \text { almost-surely } \quad \text { as } \quad n \rightarrow+\infty .
$$

We have

$$
\begin{aligned}
& \left|\mathcal{V}_{n}\left(z_{1}, z_{2}\right)\right| \\
& \leq \begin{cases}\left(1-\frac{h_{1}\left[\frac{z_{1}}{h_{1}}\right]}{z_{1}}\right)^{\alpha_{1}}\left(1-\frac{h_{y}\left[\frac{z_{2}}{h_{2}}\right]}{z_{2}}\right)^{\alpha_{2}} \sup _{(x, y) \in \mathbb{R}^{2}}|\mathbb{K}(x, y)| & \text { if } \quad\left(\alpha_{1}>0, \alpha_{2}>0\right) \\
\frac{2}{n} \sum_{k=1}^{n} K\left(\frac{X_{k}-\left[\frac{z_{1}}{h_{1}}\right] h_{1}}{h_{1}}\right) K\left(\frac{Y_{k}-\left[\frac{z_{1}}{h_{1}}\right] h_{2}}{h_{2}}\right) & \text { if } \quad\left(\alpha_{1}=0, \alpha_{2}=0\right) \\
\left(1-\frac{h_{1}\left[\frac{z_{1}}{h_{1}}\right]}{z_{1}}\right)^{\alpha_{1}} \sup _{(x, y) \in \mathbb{R}^{2}}|\mathbb{K}(x, y)| & \text { if } \quad\left(\alpha_{1}>0, \alpha_{2}=0\right) \\
\left(1-\frac{h_{2}\left[\frac{z_{2}}{h_{2}}\right]}{z_{2}}\right)^{\alpha_{2}} \sup _{(x, y) \in \mathbb{R}^{2}}|\mathbb{K}(x, y)| & \text { if } \quad\left(\alpha_{1}=0, \alpha_{2}>0\right) .\end{cases}
\end{aligned}
$$

Since

$$
\begin{aligned}
\left|\frac{\left(z_{1}-h_{1}\left[\frac{z_{1}}{h_{1}}\right]\right)\left(z_{2}-h_{2}\left[\frac{z_{2}}{h_{2}}\right]\right)-h_{1} h_{2}}{h_{1} h_{2}}\right| & =\left|\frac{z_{1} z_{2}-z_{1} h_{2}\left[\frac{z_{2}}{h_{1}}\right]-z_{2} h_{1}\left[\frac{z_{1}}{h_{1}}\right]+h_{1} h_{2}\left[\frac{z_{1}}{h_{1}}\right]\left[\frac{z_{2}}{h_{2}}\right]-h_{1} h_{2}}{h_{1} h_{2}}\right| \\
& =\left|\frac{z_{1} z_{2}}{h_{1} h_{2}}+\left[\frac{z_{1}}{h_{1}}\right]\left[\frac{z_{2}}{h_{2}}\right]-\frac{z_{1}}{h_{1}}\left[\frac{z_{2}}{h_{2}}\right]-\frac{z_{2}}{h_{2}}\left[\frac{z_{1}}{h_{1}}\right]-1\right| \\
& =\left|\frac{z_{2}}{h_{2}}\left(\frac{z_{2}}{h_{1}}-\left[\frac{z_{1}}{h_{1}}\right]\right)+\left[\frac{z_{2}}{h_{2}}\right]\left(\left[\frac{z_{1}}{h_{1}}\right]-\frac{z_{1}}{h_{1}}\right)-1\right| \\
& =\left|\left(\left[\frac{z_{2}}{h_{2}}\right]-\frac{z_{2}}{h_{2}}\right)\left(\left[\frac{z_{1}}{h_{1}}\right]-\frac{z_{1}}{h_{1}}\right)-1\right| \leq 2 .
\end{aligned}
$$

We obtain for each of the following cases: $\left(\alpha_{1}>0, \alpha_{2}>0\right),\left(\alpha_{1}>0, \alpha_{2}=0\right)$ and $\left(\alpha_{1}=\right.$ $0, \alpha_{2}>0$ )

$$
\mathcal{V}_{n}\left(z_{1}, z_{2}\right) \longrightarrow 0 \quad \text { p.s. } \quad \text { as } n \rightarrow+\infty \text {. }
$$

For $\left(\alpha_{1}=0, \alpha_{2}=0\right)$, we have also the same result since

$$
\begin{aligned}
& \left|\mathbb{E}\left[\frac{1}{n} \sum_{k=1}^{n} K\left(\frac{X_{k}-\left[\frac{z_{1}}{h_{1}}\right] h_{1}}{h_{1}}\right) K\left(\frac{Y_{k}-\left[\frac{z_{1}}{h_{1}}\right] h_{2}}{h_{2}}\right)\right]\right| \\
& =\left|h_{1} h_{2} \iint \mathbb{K}(u, v) f(x, y) d u d v\right| \\
& \leq h_{1} h_{2} \sup _{(u, v) \in \mathbb{R}^{2}} \iint|\mathbb{K}(u, v)| d u d v=O\left(h_{1} h_{2}\right) .
\end{aligned}
$$

From $\operatorname{Var}(Z)=\mathbb{E}\left(Z^{2}\right)-\mathbb{E}^{2}(Z) \leq \mathbb{E}\left(Z^{2}\right)$, we have

$$
\mathbb{E}\left[K^{2}\left(\frac{X_{k}-\left[\frac{z_{1}}{h_{1}}\right] h_{1}}{h_{1}}\right) K^{2}\left(\frac{Y_{k}-\left[\frac{z_{1}}{h_{1}}\right] h_{2}}{h_{2}}\right)\right] \leq h_{1} h_{2} \sup _{(x, y) \in \mathbb{R}^{2}} \iint\left|\mathbb{K}^{2}(u, v)\right| d u d v=O\left(h_{1} h_{2}\right) .
$$

Them by applying the strong Kolmogorov's theorem of the large numbers,

$$
\frac{2}{n} \sum_{i=1}^{n} K\left(\frac{X_{k}-\left[\frac{z_{1}}{h_{1}}\right] h_{1}}{h_{1}}\right) K\left(\frac{Y_{k}-\left[\frac{z_{1}}{h_{1}}\right] h_{2}}{h_{2}}\right) \longrightarrow 0 \quad \text { as } \quad n \rightarrow+\infty .
$$


Since $K$ being bounded, there exists $M \in \mathbb{R}_{+}$such that, $|K| \leq M$, therefore have

$$
\left|\mathcal{W}_{n}\left(z_{1}, z_{2}\right)\right| \leq\left\{\begin{array}{lll}
\left(1-\frac{h_{1}\left[\frac{z_{1}}{h_{1}}\right]}{z_{1}}\right)^{\alpha_{1}} \sup _{(x, y) \in \mathbb{R}^{2}}|\mathbb{K}(x, y)| & \text { if } \quad\left(\alpha_{1}>0, \alpha_{2}>0\right) \\
\frac{1}{n} M\left[\frac{z_{2}}{h_{2}}\right] \sum_{k=1}^{n} K\left(\frac{X_{k}-\left[\frac{z_{1}}{h_{1}}\right] h_{1}}{h_{1}}\right) & \text { if } \quad\left(\alpha_{1}=0, \alpha_{2}=0\right) \\
{\left[\frac{z_{2}}{h_{2}} \sup _{(x, y) \in \mathbb{R}^{2}}|\mathbb{K}(x, y)|\right.} & \text { if } \quad\left(\alpha_{1}>0, \alpha_{2}=0\right) \\
{\left[\frac{z_{2}}{h_{2}}\right] \sup _{(x, y) \in \mathbb{R}^{2}}|\mathbb{K}(x, y)|} & \text { if } \quad\left(\alpha_{1}=0, \alpha_{2}>0\right),
\end{array}\right.
$$

then

$$
\mathcal{W}_{n}\left(z_{1}, z_{2}\right) \longrightarrow 0 \quad \text { p.s. } \quad \text { as } n \rightarrow+\infty .
$$

For $\mathcal{U}_{n}\left(z_{1}, z_{2}\right)$ it suffices from $\mathcal{W}_{n}\left(z_{1}, z_{2}\right)$ to effect a change of role between $i$ and $j$, $x$ and $y, X$ and $Y$.

Note that

$$
\left|\frac{z_{1}-h_{1}\left[\frac{z_{1}}{h_{1}}\right]-h_{1}}{h_{1}}\right|=\left|\frac{z_{1}}{h_{1}}-\left[\frac{z_{1}}{h_{1}}\right]-1\right| \leq 1 .
$$

\section{Acknowledgment.}

The authors wish to express his thanks to the referee of this paper whose valuable comments helped to make the discussion of results more meaningful but also by pointing out several misprints and errors in simulations and to improve the writing.

\section{References}

Atkinson, A.B., 1987. On the Measurement of Poverty, Econometrica. 55 (4), 749-764.

Ciss, Y. Dia, G. and Diakhaby, A., 2014. Non-parametric estimation of income distribution and poverty index in the unidimensional context with $\alpha \in] 0,1[$. Submitted.

Dia, G., 2008. Estimation nonparamètrique de la distribution des revenus et de l'indice de pauvreté. C. R. Acad.Sci. Paris, Ser. I, 346, 907-912.

Duclos, J.-Y. Sahn, D.E. and Younger, S.D., 2006. Robust multidimensional poverty comparison. Economic Journal, 113, 943-968.

Duclos, J.-Y. Sahn, D.E. and Younger, S.D., 2006. Robust multidimensional spatial poverty comparisons in Ghana, Madagascar, and Uganda. World Bank Econ. Rev., 20(1), 91-113.

Foster, J.E., Greer, J. and Thorbecke, E., 2010. The Foster-Greer-Thorbecke (FGT) Poverty Measures: Twenty Five Years Later. Journal of Economic Inequality, 8(4), 491-524.

Foster, J. E., Greer, J. and Thorbecke, E., 1984. A class of decomposable poverty measures. Econometrica, 52, 761-776.

Foster, J.E. and Shorrocks, A.F., 1988. Poverty Orderings. Econometrica, 56, 173-177.

Foster, J.E. and Shorrocks, A.F., 1988. Poverty Orderings and Welfare Dominance. Social Choice Welfare, 5, 179-198.

Foster, J.E. and Shorrocks, A.F., 1988. Inequality and Poverty Orderings. European Economic Review, 32, 654-662.

Parzen, E., 1962. On estimation of a probability density function and mode. Annals of Mathematical Statistics, 33(3), 1065-1076. 